\title{
Identification of Torsionally Coupled Shear Buildings Models Using a Vector Parameterization
}

\author{
Antonio Concha ${ }^{1}$ and Luis Alvarez-Icaza ${ }^{2}$ \\ ${ }^{1}$ Facultad de Ingeniería Mecánica y Eléctrica, Universidad de Colima, 28400 Coquimatlán, COL, Mexico \\ ${ }^{2}$ Instituto de Ingeniería, Universidad Nacional Autónoma de México, 04510 Coyoacán, DF, Mexico \\ Correspondence should be addressed to Luis Alvarez-Icaza; alvar@pumas.iingen.unam.mx
}

Received 3 March 2016; Revised 16 May 2016; Accepted 17 May 2016

Academic Editor: Michael Todd

Copyright (C) 2016 A. Concha and L. Alvarez-Icaza. This is an open access article distributed under the Creative Commons Attribution License, which permits unrestricted use, distribution, and reproduction in any medium, provided the original work is properly cited.

\begin{abstract}
A methodology to estimate the shear model of seismically excited, torsionally coupled buildings using acceleration measurements of the ground and floors is presented. A vector parameterization that considers Rayleigh damping for the building is introduced that allows identifying the stiffness/mass and damping/mass ratios of the structure, as well as their eccentricities and radii of gyration. This parameterization has the advantage that its number of parameters is smaller than that obtained with matrix parameterizations or when Rayleigh damping is not used. Thus, the number of spectral components of the excitation signal required to identity the structural parameters is reduced. To deal with constant disturbances and measurement noise that corrupt acceleration measurements, Linear Integral Filters are used that guarantee elimination of constant disturbances and attenuation of noise.
\end{abstract}

\section{Introduction}

The parameter identification of torsionally coupled shear building models has been a topic of interest in the last three decades [1-13]. Its identification is important because it allows verifying the structural health, or designing control law techniques that attenuate the vibration of the building when it is excited by external forces as earthquakes or wind [14-16]. This kind of model more closely approximates a shear building than the planar frame model, which is widely used in the literature [17-19]. The reason is that most buildings present torsional movements under purely translational excitations since their centers of resistance and mass do not generally coincide [20]. Although in some identification schemes buildings are modeled as two independent planar frames where torsional motion is neglected, the focus in this paper is in recovering the model of buildings where the centers of mass and the centers of torsion in each floor do not coincide and, therefore, significant torsional motions are expected. There are significant differences between the responses of these two approaches, whose comparison is beyond the scope of this paper.
References [1-8] propose techniques to estimate the modal parameters of torsionally coupled buildings using acceleration measurements. Li and Mau [1] present a methodology that identity the modal parameters by minimizing the error between the measured accelerations and the ones predicted by the solution of the Duhamel integral. Ueng et al. [2], Lin et al. [3], and Nayeri et al. [4] obtain the parameters of a structure excited by means of ambient vibrations; [2,3] combine an extended decrement random method and the Ibrahim Time Domain estimation technique, whereas [4] identifies a full-scale 17-story building using the NExT/ERA (Natural Excitation Technique in conjunction with the Eigensystem Realization Algorithm) and a time domain identification technique for chain-like MDOF systems. In $[5,6]$, identification techniques that employ the Eigensystem Realization Algorithm (ERA) in order to generate a building state space model are proposed. Hegde and Sinha [6] use only acceleration measurements of the top and first floor levels, but their method is applied only to structures whose masses and eccentricities are equal for all stories. Antonacci et al. [7] identify the modal parameters by means of the following methods: Enhanced Frequency Domain Decomposition 
(EFFD), ERA, Stochastic Subspace Identification (SSI), and Time-Frequency Instantaneous Estimators (TFIE). The algorithms in $[8,9]$ estimate the stiffness matrix of torsionally coupled buildings; Torkamani and Ahmadi [8] firstly identify the natural frequencies and modal shapes by means of the Fourier spectra of the acceleration of the floors; then, a parameter identification technique, which assumes knowledge of the building floors masses, is used to obtain the stiffness matrix. Omrani et al. [9] obtain the stiffness matrix from a methodology that uses the structure response to ambient excitation and the knowledge of all the masses and eccentricities of the floors. Wang et al. [10] proposed a procedure that combines the identification technique SRIM (System Realization using Information Matrix) and a damage index in order to estimate the damage of torsional coupled buildings. Angeles-Cervantes and Alvarez-Icaza [13] propose a technique that combines the online Least Squares Method with a parametrization of the building, which in the sequel will be called matrix parameterization, that is used for estimating the complete matrices $\mathbb{M}^{-1} \mathbb{K}$ and $\mathbb{M}^{-1} \mathbb{C}$ of the structure, where $\mathbb{M}, \mathbb{K}$, and $\mathbb{C}$ are the mass, stiffness, and damping matrices, respectively; however, the identified matrices are overparameterized and, as a consequence the stiffness/mass and damping/mass ratios of the structure cannot be uniquely identified; in addition, with this parameterization, the zeros entries of the matrices $\mathbb{M}^{-1} \mathbb{K}$ and $\mathbb{M}^{-1} \mathbb{C}$ are also identified, which greatly increases the number of parameters to be estimated. Finally, $[11,12]$ propose an identification approach, based on the unscented Kalman filter, that identifies the stiffness and damping parameters of a torsionally coupled building, as well as the Bouc-Wen model parameters that represent the hysteretic response of each lateral load resisting elements of the structure; the algorithm in [12] is an extension of [11] and also estimates the mass eccentricities of the building; the unscented Kalman filter, proposed in these two references, uses only acceleration measurements and also estimates the velocity and displacement of the structure, thus avoiding the numerical integration of the acceleration measurements, which leads to velocity and displacement histories that drift away linearly and quadratically with the time, respectively [21]. The approach in [11] attenuates measurement noise; however, the constant disturbance voltage in accelerometers output cannot be eliminated by a Kalman filter. The usual approach to eliminate these constant disturbances is the offline processing of the accelerometer signals.

This paper presents an identification technique that estimates the parameters of a torsionally coupled building model, which exhibits torsionally movements under purely translational excitations due to a seismic event. A vector parameterization of the building is proposed that has the following characteristics: (1) it assumes that the structure has classical Rayleigh damping and (2) it contains a vector whose entries depend on the building stiffness/mass ratios, which are estimated using accelerations measurements of the ground and floors. Once that these ratios are identified, it is possible to estimate the building damping/mass ratios and the modal parameters; additionally, it is also possible to identify the radii of gyration and eccentricities of the floors and the stiffness, damping, and mass matrices of the structure.
In contrast to the estimation methods in $[6,8,9]$, the proposed technique does not assume that the eccentricities of all the floors are in the same direction or that all the floor masses are equal or known. The proposed vector parameterization is combined with the offline or with the online Least Squares Method (LSM) and can be used for estimating the complete model of the structure if all the floors are instrumented or be employed for identifying a reduced model of the building, if only some floors are equipped with accelerometers.

Assuming Rayleigh damping for the structure permits reducing the number of parameters contained in the proposed vector parameterization, since it is composed only of stiffness/mass ratios instead of stiffness/mass and damping/ratios as in the matrix parameterization [13] or the work of Omrani et al. [12]. The number of parameters contained in the vector parameterization is thus reduced with the positive impact on the (1) reduction of the computational effort that allows implementing the online LSM and (2) the reduction of the spectral richness of the excitation signal required to uniquely estimate the structural parameters.

This paper extends the use of the Linear Integral Filters (LIF), first introduced in [19], to eliminate constant disturbances in the acceleration measurements and to attenuate measurement noise, to the parameter estimation of torsionally coupled building models, a more challenging problem when real-time estimation is desired, as the number of parameters involved in the torsion based model greatly increases.

The paper is organized as follows. Section 2 describes the model of a torsionally coupled multistory building. Section 3 presents the proposed vector parameterization and introduces the LIF. Section 4 shows both the offline and the online LSMs employed to estimate the stiffness/mass ratios and describes the methodology that allows estimating the damping/mass ratios, eccentricities, radii of gyration, and the mass, stiffness, and damping matrices of the structure. Experimental results in a five-story torsional building obtained with the vector parameterization and the LSM are presented in Section 5; three cases are considered, the first where all the floors are instrumented, the second where only the first, third, and fifth stories are equipped with accelerometers, and the third where only the first and the top floors are instrumented. Finally, Section 6 establishes the conclusions of this paper.

\section{Mathematical Model of a Torsionally Coupled Shear Building}

Figure 1 shows a torsionally coupled shear building that is seismically excited, where the centers of mass and centers of resistance of the floors do not lie on one vertical axis. The building model is defined as [2, 10, 22, 23]

$$
\mathbb{M}\left(\ddot{U}+\ddot{U}_{g}\right)+\mathbb{C} \dot{U}+\mathbb{K} U=O_{3 n \times 1}
$$

where $\mathbb{M}, \mathbb{K}$, and $\mathbb{C}$ are, respectively, the mass, stiffness, and damping matrices. Moreover, the variable $O_{v \times w}$ denotes a zero matrix of size $v \times w$, and $\ddot{U}_{g}$ represents the absolute 
ground acceleration induced by an earthquake, which is given by

$$
\begin{aligned}
\ddot{U}_{g} & =\left[\ddot{U}_{g x}^{T}, \ddot{U}_{g y}^{T}, O_{1 \times n}\right]^{T}, \\
\ddot{U}_{g x} & =[1, \ldots, 1]^{T} \ddot{u}_{g x} \in R^{n \times 1}, \\
\ddot{U}_{g y} & =[1, \ldots, 1]^{T} \ddot{u}_{g y} \in R^{n \times 1},
\end{aligned}
$$

where $n$ is the total number of floors and the terms $\ddot{u}_{g x}$ and $\ddot{u}_{g y}$ are the ground accelerations in the $x$ and $y$ directions, respectively. Furthermore, vector $U$ is defined as

$$
U=\left[U_{x}^{T}, U_{y}^{T}, U_{\theta}^{T}\right]^{T},
$$

where $U_{i} \in R^{n \times 1}, i=x, y, \theta$, is the displacement vector in the $i$ th coordinate and $\dot{U}$ and $\ddot{U}$ are the first and the second time derivatives of $U$. Vectors $U_{i}, i=x, y, \theta$, have the following structure:

$$
\begin{aligned}
& U_{x}=\left[x_{1}, x_{2}, \ldots, x_{n}\right]^{T}, \\
& U_{y}=\left[y_{1}, y_{2}, \ldots, y_{n}\right]^{T}, \\
& U_{\theta}=\left[\theta_{1}, \theta_{2}, \ldots, \theta_{n}\right]^{T} .
\end{aligned}
$$

Note from Figure 1 that each story has three displacements, two in the $x$ and $y$ directions relative to the ground and a rotation of its center of mass about the vertical axis. Moreover, the terms $\mathrm{cm}$ and $\mathrm{cr}$ in this figure denote the center of mass and the center of resistance of the corresponding story, respectively.

\section{Mass matrix $\mathbb{M}$ is}

$$
\mathbb{M}=\mathbb{M}^{T}=\left[\begin{array}{ccc}
m_{f} & O_{n \times n} & O_{n \times n} \\
O_{n \times n} & m_{f} & O_{n \times n} \\
O_{n \times n} & O_{n \times n} & I_{f}
\end{array}\right] \in R^{3 n \times 3 n} .
$$

The entries $m_{f}$ and $I_{f}$ of $\mathbb{M}$ are, respectively, diagonal matrices that contain the masses of the stories and the moment of inertia of them about the vertical axis that pass through their center of mass. These matrices are given by

$$
\begin{aligned}
m_{f} & =\operatorname{diag}\left(m_{1}, m_{2}, m_{3}, m_{4}, \ldots, m_{n}\right), \\
I_{f} & =\operatorname{diag}\left(I_{1}, I_{2}, I_{3}, I_{4}, \ldots, I_{n}\right) \\
& =\operatorname{diag}\left(m_{1} r_{1}^{2}, m_{2} r_{2}^{2}, m_{3} r_{3}^{2}, m_{4} r_{4}^{2}, \ldots, m_{n} r_{n}^{2}\right),
\end{aligned}
$$

where $\operatorname{diag}(p)$ is a square diagonal matrix with the elements of the vector $p$ on the main diagonal, $m_{i}$ and $I_{i}, i=1,2, \ldots, n$, are, respectively, the lumped mass and the moment of inertia at floor $i$; moreover, $r_{i}$ is the radius of gyration of the $i$ th floor around the vertical axis passing through its centers of mass. On the other hand, the structure of the stiffness matrix $\mathbb{K}$ is the following:

$$
\mathbb{K}=\mathbb{K}^{T}=\left[\begin{array}{lll}
K_{X X} & O_{n \times n} & K_{X \Theta} \\
O_{n \times n} & K_{Y Y} & K_{Y \Theta} \\
K_{X \Theta}^{T} & K_{Y \Theta}^{T} & K_{\Theta \Theta}
\end{array}\right] \in R^{3 n \times 3 n},
$$

where the submatrices of $\mathbb{K}$ have a size of $n \times n$ and are defined as

$$
\begin{aligned}
& K_{X X}=\left[\begin{array}{ccccc}
K_{x 1}+K_{x 2} & -K_{x 2} & 0 & \cdots & 0 \\
-K_{x 2} & K_{x 2}+K_{x 3} & -K_{x 3} & \cdots & 0 \\
0 & -K_{x 3} & K_{x 3}+K_{x 4} & \cdots & 0 \\
\vdots & \vdots & \vdots & \ddots & \vdots \\
0 & 0 & 0 & \cdots & K_{x n}
\end{array}\right] \\
& K_{Y Y}=\left[\begin{array}{ccccc}
K_{y 1}+K_{y 2} & -K_{y 2} & 0 & \cdots & 0 \\
-K_{y 2} & K_{y 2}+K_{y 3} & -K_{y 3} & \cdots & 0 \\
0 & -K_{y 3} & K_{y 3}+K_{y 4} & \cdots & 0 \\
\vdots & \vdots & \vdots & \ddots & \vdots \\
0 & 0 & 0 & \cdots & K_{y n}
\end{array}\right] \text {, } \\
& K_{X \Theta}=-\left[\begin{array}{ccccc}
e_{y 1,1} K_{x 1}+e_{y 1,2} K_{x 2} & -e_{y 2,2} K_{x 2} & 0 & \cdots & 0 \\
-e_{y 1,2} K_{x 2} & e_{y 2,2} K_{x 2}+e_{y 2,3} K_{x 3} & -e_{y 3,3} K_{x 3} & \cdots & 0 \\
0 & -e_{y 2,3} K_{x 3} & e_{y 3,3} K_{x 3}+e_{y 3,4} K_{x 4} & \cdots & 0 \\
\vdots & \vdots & \vdots & \ddots & \vdots \\
0 & 0 & 0 & \cdots & e_{y n, n} K_{x n}
\end{array}\right] \text {, }
\end{aligned}
$$




$$
\begin{aligned}
& K_{Y \Theta}=\left[\begin{array}{ccccc}
e_{x 1,1} K_{y 1}+e_{x 1,2} K_{y 2} & -e_{x 2,2} K_{y 2} & 0 & \cdots & 0 \\
-e_{x 1,2} K_{y 2} & e_{x 2,2} K_{y 2}+e_{x 2,3} K_{y 3} & -e_{x 3,3} K_{y 3} & \cdots & 0 \\
0 & -e_{x 2,3} K_{y 3} & e_{x 3,3} K_{y 3}+e_{x 3,4} K_{y 4} & \cdots & 0 \\
\vdots & \vdots & \vdots & \ddots & \vdots \\
0 & 0 & 0 & \cdots & e_{x n, n} K_{y n}
\end{array}\right], \\
& K_{\Theta \Theta}=\left[\begin{array}{ccccc}
K_{91} & -K_{92} & 0 & \cdots & 0 \\
-K_{92} & K_{93} & -K_{94} & \cdots & 0 \\
0 & -K_{94} & K_{95} & \cdots & 0 \\
\vdots & \vdots & \vdots & \ddots & \vdots \\
0 & 0 & 0 & \cdots & K_{\vartheta(2 n-1)}
\end{array}\right] \\
& K_{\vartheta 1}=K_{\theta 1}+K_{\theta 2}+K_{x 1} e_{y 1,1}^{2}+K_{x 2} e_{y 1,2}^{2}+K_{y 1} e_{x 1,1}^{2}+K_{y 2} e_{x 1,2}^{2} \text {, } \\
& K_{92}=K_{\theta 2}+K_{x 2} e_{y 1,2} e_{y 2,2}+K_{y 2} e_{x 1,2} e_{x 2,2} \\
& K_{93}=K_{\theta 2}+K_{\theta 3}+K_{x 2} e_{y 2,2}^{2}+K_{x 3} e_{y 2,3}^{2}+K_{y 2} e_{x 2,2}^{2}+K_{y 3} e_{x 2,3}^{2} \text {, } \\
& K_{94}=K_{\theta 3}+K_{x 3} e_{y 2,3} e_{y 3,3}+K_{y 3} e_{x 2,3} e_{x 3,3} \text {, } \\
& K_{95}=K_{\theta 3}+K_{\theta 4}+K_{x 3} e_{y 3,3}^{2}+K_{x 4} e_{y 3,4}^{2}+K_{y 3} e_{x 3,3}^{2}+K_{y 4} e_{x 3,4}^{2}, \\
& K_{\vartheta(2 n-1)}=K_{\theta n}+K_{x n} e_{y n, n}^{2}+K_{y n} e_{x n, n}^{2} \text {. }
\end{aligned}
$$

The elements $K_{x i}$ and $K_{y i}$ given in (8)-(11) are the equivalent stiffness between floors $i$ and $i-1$ along the $x$-and $y$-axes, respectively; $K_{\theta i}$ in (13) is the torsional stiffness of the $i$ th floor about a vertical axis at its center of resistance. Moreover, $e_{v i, i}$ and $e_{v i, i+1}$ with $v=x, y$ denote the static eccentricities in $v$ axis at floor $i$ with respect to stories $i$ and $i+1$, respectively.

The damping $\mathbb{C}$ of building (1) will be represented by a Rayleigh damping matrix, which is given by

$$
\mathbb{C}=a_{0} \mathbb{M}+a_{1} \mathbb{K}
$$

where $a_{0}$ and $a_{1}$ are constants computed by the next equation [17]:

$$
\frac{1}{2}\left[\begin{array}{cc}
\frac{1}{\omega_{i}} & \omega_{i} \\
\frac{1}{\omega_{j}} & \omega_{j}
\end{array}\right]\left[\begin{array}{l}
a_{0} \\
a_{1}
\end{array}\right]=\left[\begin{array}{l}
\zeta_{i} \\
\zeta_{j}
\end{array}\right],
$$

where $\zeta_{v}$ and $\omega_{v}, v=i, j$, are the damping ratio and natural frequency of the $v$ th mode of the structure.

2.1. Assumptions. In order to apply the proposed identification technique, assume the following:

(A1) Initial conditions $U(0)$ and $\dot{U}(0)$ are zero, which is reasonable since the structure is at rest before an earthquake.

(A2) Three acceleration measurements are available for each floor, two in the $x$ direction and one in the $y$ direction, or vice versa. Thus, with these measurements it is possible to obtain the acceleration of the centers of mass (CM) of the floors in the $x$ and $y$ directions, as well as their angular accelerations [3, 13]. Moreover, the ground accelerations in the $x$ and $y$ directions are also accessible.

Remark 1. In order to recover the acceleration of the CM of each floor with three accelerometers, it is necessary to determine the floor's CM position. This point can be deduced from the geometry of the story and the position and weight of its columns. Note that the column weight depends on its materials and dimensions.

Remark 2. The building can also be identified when only the three acceleration measurements of $n_{r}$ floors are available, where $n_{r}<n$. In this case the torsional building is considered as a structure with $n_{r}$ floors, where every condensed floor of the building will consist of the instrumented level and all the floors beneath it that are not instrumented:

(A3) Estimates $\widehat{\omega}_{i \star}$ and $\widehat{\omega}_{j \star}$ of two natural frequencies $\omega_{i}$ and $\omega_{j}$ of the structure are available. Note that these estimated frequencies can be extracted from Fourier spectra of the acceleration responses due to ambient or force excitations of the building [24].

(A4) Estimates $\widehat{\zeta}_{i \star}$ and $\widehat{\zeta}_{j \star}$ of the damping ratios for the $i$ th and $j$ th modes are also accessible. These estimates can be obtained using the recommended damping 


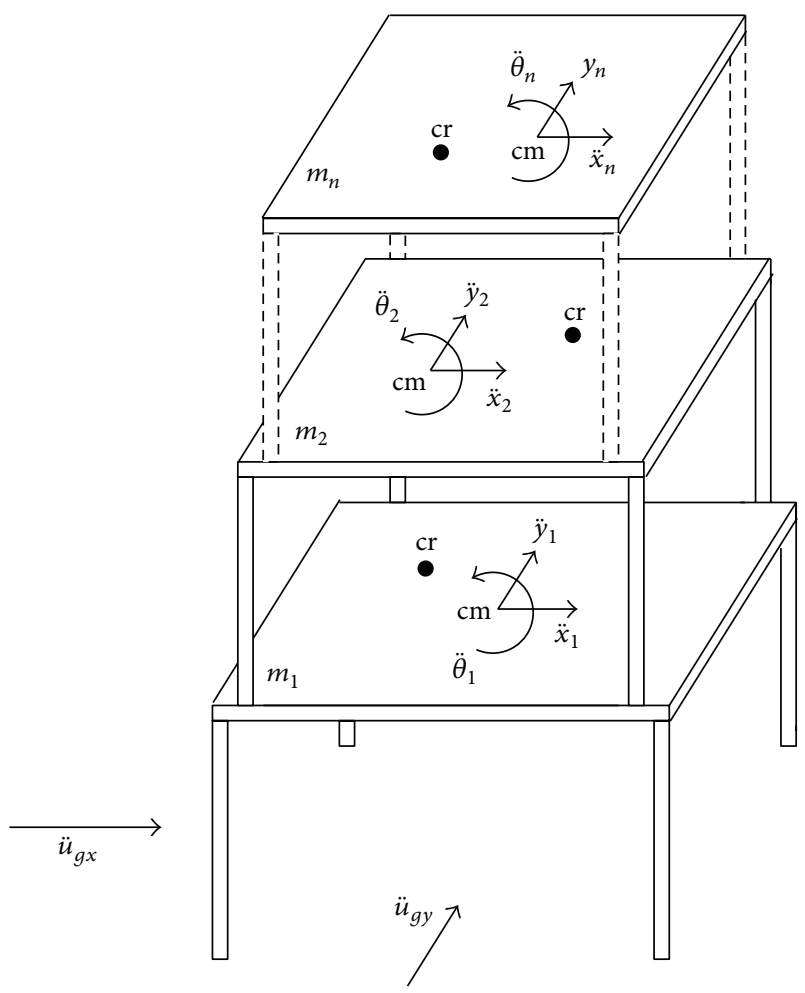

FIGURE 1: Torsionally coupled building with $n$ floors.

values for buildings, which depend on the structure materials and can be found in Table 11.2.1 from [17]. The next values of damping are recommended, $1-2 \%$ for steel buildings and 3-5\% for reinforced concrete buildings [18]. This assumption allows computing the parameters $a_{0}$ and $a_{1}$ in (14) and (15) as follows:

$$
\begin{aligned}
& a_{0}=2\left(\frac{\widehat{\omega}_{i \star}^{2} \widehat{\omega}_{j \star} \widehat{\zeta}_{j \star}-\widehat{\omega}_{j \star}^{2} \widehat{\omega}_{i \star} \widehat{\zeta}_{i \star}}{\widehat{\omega}_{i \star}^{2}-\widehat{\omega}_{j \star}^{2}}\right), \\
& a_{1}=2\left(\frac{\widehat{\omega}_{i \star} \widehat{\zeta}_{i \star}-\widehat{\omega}_{j \star} \widehat{\zeta}_{j \star}}{\widehat{\omega}_{i \star}^{2}-\widehat{\omega}_{j \star}^{2}}\right) .
\end{aligned}
$$

Remark 3. The proposed identification method does not need the exact knowledge of the natural frequencies $\omega_{i}$ and $\omega_{j}$ used to compute the parameters $a_{0}$ and $a_{1}$. This fact is reflected in Section 5.1, where the proposed method estimates practically the same model in the next three cases: (a) $\widehat{\omega}_{i \star}=\omega_{i}, \widehat{\omega}_{j \star}=\omega_{j}$; (b) $\widehat{\omega}_{i \star}=0.5 \omega_{i}, \widehat{\omega}_{j \star}=0.5 \omega_{j}$; and (c) $\widehat{\omega}_{i \star}=1.5 \omega_{i}, \widehat{\omega}_{j \star}=1.5 \omega_{j}$.

(A5) All the acceleration measurements are corrupted by constant disturbances and measurement noise; that is,

$$
\begin{aligned}
& \ddot{u}_{g x m}=\ddot{u}_{g x}+d_{g x}+\xi_{g x}, \\
& \ddot{u}_{g y m}=\ddot{u}_{g y}+d_{g y}+\xi_{g y},
\end{aligned}
$$

$$
\begin{aligned}
& \ddot{U}_{x m}=\ddot{U}_{x}+D_{x}+\sigma_{x}, \\
& \ddot{U}_{y m}=\ddot{U}_{y}+D_{y}+\sigma_{y}, \\
& \ddot{U}_{\theta m}=\ddot{U}_{\theta}+D_{\theta}+\sigma_{\theta} .
\end{aligned}
$$

Terms $\ddot{u}_{\text {gxm }}$ and $\ddot{u}_{\text {gym }}$ are the measured accelerations of the ground in the $x$ and $y$ directions, respectively. Variables $\ddot{U}_{x m}=\left[\ddot{x}_{1 m}, \ldots, \ddot{x}_{n m}\right]^{T}, \ddot{U}_{y m}=$ $\left[\ddot{y}_{1 m}, \ldots, \ddot{y}_{n m}\right]^{T}$, and $\ddot{U}_{\theta m}=\left[\ddot{\theta}_{1 m}, \ldots, \ddot{\theta}_{n m}\right]^{T}$ are vectors that contain the measured accelerations of the centers of mass of the floors in the $x, y$, and $\theta$ directions, respectively. Moreover, the elements of the vectors $D_{x}=\left[d_{x 1}, \ldots, d_{x n}\right]^{T}, D_{y}=\left[d_{y 1}, \ldots, d_{y n}\right]^{T}, D_{\theta}=$ $\left[d_{\theta 1}, \ldots, d_{\theta n}\right]^{T}$, and $d_{g x}$ and $d_{g y}$ are constant disturbances. Finally, the entries of $\sigma_{x}=\left[\xi_{x 1}, \ldots, \xi_{x n}\right]^{T}$, $\sigma_{y}=\left[\xi_{y 1}, \ldots, \xi_{y n}\right]^{T}, \sigma_{\theta}=\left[\xi_{\theta 1}, \ldots, \xi_{\theta n}\right]^{T}$, and $\xi_{g x}$ and $\xi_{\text {gy }}$ are measurement noises.

Define the following vectors:

$$
\begin{aligned}
\ddot{U}_{g x m} & =[1, \ldots, 1]^{T} \ddot{u}_{g x m}, \\
\ddot{U}_{g y m} & =[1, \ldots, 1]^{T} \ddot{u}_{g y m}, \\
D_{g x} & =[1, \ldots, 1]^{T} d_{g x}, \\
D_{g y} & =[1, \ldots, 1]^{T} d_{g y}, \\
\sigma_{g x} & =[1, \ldots, 1]^{T} \xi_{g x}, \\
\sigma_{g y} & =[1, \ldots, 1]^{T} \xi_{g y}, \\
\ddot{U}_{g m} & =\left[\ddot{U}_{g x m}^{T}, \ddot{U}_{g y m}^{T}, O_{1 \times n}\right]^{T}, \\
D_{g} & =\left[D_{g x}^{T}, D_{g y}^{T}, O_{1 \times n}\right]^{T}, \\
\sigma_{g} & =\left[\sigma_{g x}^{T}, \sigma_{g y}^{T}, O_{1 \times n}\right]^{T} .
\end{aligned}
$$

Substituting the signals $\ddot{u}_{g x}$ and $\ddot{u}_{g y}$ (17) in (2) and using the equations in (19) lead to

$$
\ddot{U}_{g}=\ddot{U}_{g m}-D_{g}-\sigma_{g} .
$$

Now define

$$
\begin{aligned}
\ddot{U}_{m} & =\left[\ddot{U}_{x m}^{T}, \ddot{U}_{y m}^{T}, \ddot{U}_{\theta m}^{T}\right]^{T}, \\
D & =\left[D_{x}^{T}, D_{y}^{T}, D_{\theta}^{T}\right]^{T}, \\
\sigma & =\left[\sigma_{x}^{T}, \sigma_{y}^{T}, \sigma_{\theta}^{T}\right]^{T} .
\end{aligned}
$$

Using this definition and (18) produces

$$
\ddot{U}=\ddot{U}_{m}-D-\sigma,
$$

where $\ddot{U}=\left[\ddot{U}_{x}^{T}, \ddot{U}_{y}^{T}, \ddot{U}_{\theta}^{T}\right]^{T}$. 


\section{Vector Parameterization}

Expression (1) is equivalent to

$$
\ddot{U}+\ddot{U}_{g}=-\mathbb{M}^{-1} \mathbb{C} \dot{U}-\mathbb{M}^{-1} \mathbb{K} U .
$$

Substituting $\mathbb{C}$ from (14) into (23) gives

$$
\ddot{U}+\ddot{U}_{g}+a_{0} \dot{U}=-a_{1} \mathbb{M}^{-1} \mathbb{K} \dot{U}-\mathbb{M}^{-1} \mathbb{K} U .
$$

The product $-\mathbb{M}^{-1} \mathbb{K} U$ in (24) can be parameterized as

$$
-\mathbb{M}^{-1} \mathbb{K} U=W \Theta_{K}
$$

where the vector $\Theta_{K}$ contains the stiffness/mass ratios of the structure and is defined in (A.1); moreover,

$$
\begin{aligned}
W & =\left[\begin{array}{ccccccc}
w_{x} & w_{\theta} & O_{z} & O_{v} & O_{z} & O_{z} & O_{v} \\
O_{z} & O_{v} & w_{y} & -w_{\theta} & O_{z} & O_{z} & O_{v} \\
O_{z} & O_{v} & O_{z} & O_{v} & -w_{x} & w_{y} & -w_{\theta}
\end{array}\right] \in R^{3 n \times(17 n-10)}, \quad z=n \times(2 n-1), v=n \times(3 n-2), \\
w_{x}= & -\left[\begin{array}{cccccccc}
x_{1} & x_{1}-x_{2} & 0 & 0 & 0 & 0 & \cdots & 0 \\
0 & 0 & x_{2}-x_{1} & x_{2}-x_{3} & 0 & 0 & \cdots & 0 \\
0 & 0 & 0 & 0 & x_{3}-x_{2} & x_{3}-x_{4} & \cdots & 0 \\
\vdots & \vdots & \vdots & \vdots & \vdots & \vdots & & \vdots \\
0 & 0 & 0 & 0 & 0 & 0 & \cdots & x_{n}-x_{n-1}
\end{array}\right] \in R^{n \times(2 n-1)}, \\
w_{\theta}= & {\left[\begin{array}{cccccccc}
\theta_{1} & -\theta_{2} & 0 & 0 & 0 & \cdots & 0 & 0 \\
0 & 0 & -\theta_{1} & \theta_{2} & -\theta_{3} & \cdots & 0 & 0 \\
\vdots & \vdots & \vdots & \vdots & \vdots & \ddots & \vdots & \vdots \\
0 & 0 & 0 & 0 & 0 & \cdots & -\theta_{n-1} & \theta_{n}
\end{array}\right] \in R^{n \times(3 n-2)} . }
\end{aligned}
$$

The matrix $w_{y}$ has the same structure as $w_{x}$ and uses the signals $y_{1}, \ldots, y_{n}$.

Similarly, the product $-a_{1} \mathbb{M}^{-1} \mathbb{K} \dot{U}$ in (24) is parameterized as follows:

$$
-a_{1} \mathbb{M}^{-1} \mathbb{K} \dot{U}=a_{1} \dot{W} \Theta_{K},
$$

where $\dot{W}$ is the time derivative of $W(26)$.

Equalities (25) and (29) allow writing expression (24) as

$$
\ddot{U}+\ddot{U}_{g}+a_{0} \dot{U}=\left(a_{1} \dot{W}+W\right) \Theta_{K} .
$$

The Laplace transform of (30) is given by

$$
\mathscr{L}\left[\ddot{U}+\ddot{U}_{g}\right]+a_{0} s U=\left(a_{1} s \mathscr{L}[W]+\mathscr{L}[W]\right) \Theta_{K} \cdot
$$

Multiplying (31) by $s^{3}$ in order to obtain only acceleration signals leads to

$$
\begin{aligned}
s^{3} \mathscr{L} & {\left[\ddot{U}+\ddot{U}_{g}\right]+a_{0} s^{4} \mathscr{L}[U] } \\
& =\left(a_{1} s^{4} \mathscr{L}[W]+s^{3} \mathscr{L}[W]\right) \Theta_{K} .
\end{aligned}
$$

Equation (32) is equivalent to

$$
\begin{aligned}
s^{3} \mathscr{L} & {\left[\ddot{U}+\ddot{U}_{g}\right]+a_{0} s^{2} \mathscr{L}[\ddot{U}] } \\
& =\left(a_{1} s^{2} \mathscr{L}[\ddot{W}]+s \mathscr{L}[\ddot{W}]\right) \Theta_{K},
\end{aligned}
$$

where $\ddot{W}$ is the second time derivative of $W$ in (26).
Substituting the entries of the vector $\ddot{U}$ from (22) into $\ddot{W}$ leads to

$$
\ddot{W}=\ddot{W}_{m}-\gamma-v \text {. }
$$

The matrix $\ddot{W}_{m}$ has the same structure as $\ddot{W}$ and is composed of measured accelerations; moreover,

$$
\begin{aligned}
\gamma & =\left[\begin{array}{ccccccc}
\gamma_{x} & \gamma_{\theta} & O_{z} & O_{v} & O_{z} & O_{z} & O_{v} \\
O_{z} & O_{v} & \gamma_{y} & -\gamma_{\theta} & O_{z} & O_{z} & O_{v} \\
O_{z} & O_{v} & O_{z} & O_{v} & -\gamma_{x} & \gamma_{y} & -\gamma_{\theta}
\end{array}\right] \\
& \in R^{3 n \times(17 n-10)}, \\
v & =\left[\begin{array}{ccccccc}
v_{x} & -v_{\theta} & O_{z} & O_{z} & O_{z} & O_{z} & O_{z} \\
O_{z} & O_{z} & v_{y} & v_{\theta} & O_{z} & O_{z} & O_{z} \\
O_{z} & O_{z} & O_{z} & O_{z} & -v_{x} & v_{y} & v_{\theta}
\end{array}\right] \in R^{3 n \times(17 n-10)},
\end{aligned}
$$

where $\gamma_{x}, \gamma_{y}$, and $\gamma_{\theta}$ in (35) are obtained by replacing the signals $x_{i}, y_{i}$, and $\theta_{i}$ with the disturbances $d_{x i}, d_{y i}$, and $d_{\theta i}$ in the submatrices $w_{x}, w_{y}$, and $w_{\theta}$ given in (26). Similarly, $v_{x}$, $v_{y}$, and $v_{\theta}$ are derived by substituting $x_{i}, y_{i}$, and $\theta_{i}$ with the noises $\xi_{x i}, \xi_{y i}$, and $\xi_{\theta i}$ in the submatrices $w_{x}, w_{y}$, and $w_{\theta}$. 
Replacing $\ddot{U}_{g}(20), \ddot{U}$ (22), and $\ddot{W}$ (34) into (33) gives

$$
\begin{aligned}
& s^{3} Y_{m}(s)+a_{0} s^{2} \mathscr{L}\left[\ddot{U}_{m}\right] \\
& \quad=\left(a_{1} s^{2} \mathscr{L}\left[\ddot{W}_{m}\right]+s \mathscr{L}\left[\ddot{W}_{m}\right]\right) \Theta_{K}+R(s)+\Lambda(s), \\
& Y_{m}(s)=\mathscr{L}\left[\ddot{U}_{m}+\ddot{U}_{g m}\right], \\
& R(s)=s^{2}\left(D+D_{g}\right)+a_{0} s D-\gamma \Theta_{K}\left(a_{1} s+1\right),
\end{aligned}
$$

$\Lambda(s)$

$$
\begin{aligned}
= & s^{3}\left[\Sigma_{g}(s)+\Sigma(s)\right]+a_{0} s^{2} \Sigma(s) \\
& -\Upsilon(s) \Theta_{K}\left(a_{1} s^{2}+s\right)
\end{aligned}
$$

with $\Sigma(s)=\mathscr{L}[\sigma], \Sigma_{g}(s)=\mathscr{L}\left[\sigma_{g}\right]$, and $\Upsilon(s)=\mathscr{L}[v]$. Note that variables $R(s)$ and $\Lambda(s)$ depend on the constant disturbances and the measured noise, respectively.

Equation (37) can be written in the time domain as

$$
\begin{aligned}
y_{m}^{(3)}(t)+a_{0} \ddot{U}_{m}^{(2)}(t)= & \left(a_{1} \ddot{W}_{m}^{(2)}(t)+\ddot{W}_{m}^{(1)}(t)\right) \Theta_{K} \\
& +r(t)+\lambda(t),
\end{aligned}
$$

where the superscript $(i), i=1,2$, represents the $i$ th time derivative, $r(t)=\mathscr{L}^{-1}[R(s)]$, and $\lambda(t)=\mathscr{L}^{-1}[\Lambda(s)]$.

In order to avoid the use of the derivatives of the measured signals $\ddot{U}_{m}, \ddot{U}_{g m}$, and $\ddot{W}_{m}$ and to attenuate the term $\lambda$ that depends on the measurement noise, (41) is integrated five times over finite time periods using Linear Integral Filters (LIF). Before carrying out this integration, it is useful to define the next operator [25]:

$$
\begin{aligned}
& I_{n}\{\rho(t)\} \\
& \quad=\frac{1}{\delta^{n-1}} \int_{t-\delta}^{t} \int_{\tau_{1}-\delta}^{\tau_{1}} \ldots \int_{\tau_{n-1}-\delta}^{\tau_{n-1}} \rho\left(\tau_{n}\right) d \tau_{n} \cdots d \tau_{1},
\end{aligned}
$$

where $n$ is the number of integrations over finite time periods and $\delta$ is the integration time period defined as $\delta=\hbar T_{s}$, with $\hbar>0$ and $T_{s}$ as the sampling period. The Laplace transform of (42) is given by

$$
\mathscr{L}\left[I_{n}\{\rho(t)\}\right]=\frac{1}{\delta^{n-1}}\left(\frac{1-e^{-\delta s}}{s}\right)^{n} \mathscr{L}[\rho(t)] .
$$

Applying the operator $I_{5}[\cdot]$ to (41) leads to

$$
\begin{aligned}
\chi(t)= & \Phi_{K}(t) \Theta_{K}+I_{5}[r(t)]+I_{5}[\lambda(t)], \\
\chi(t)= & \frac{1}{\delta^{3}} I_{2}\left\{\sum_{j=0}^{3}\left(\begin{array}{l}
3 \\
j
\end{array}\right)(-1)^{j} y_{m}(t-j \delta)\right\} \\
& +\frac{a_{0}}{\delta^{2}} I_{3}\left\{\sum_{j=0}^{2}\left(\begin{array}{l}
2 \\
j
\end{array}\right)(-1)^{j} \ddot{U}_{m}(t-j \delta)\right\},
\end{aligned}
$$

$$
\begin{aligned}
\Phi_{K}(t)= & \frac{a_{1}}{\delta^{2}} I_{3}\left\{\sum_{j=0}^{2}\left(\begin{array}{l}
2 \\
j
\end{array}\right)(-1)^{j} \ddot{W}_{m}(t-j \delta)\right\} \\
& +\frac{1}{\delta} I_{4}\left\{\sum_{j=0}^{1}\left(\begin{array}{l}
1 \\
j
\end{array}\right)(-1)^{j} \ddot{W}_{m}(t-j \delta)\right\},
\end{aligned}
$$

where the expression $\left(\begin{array}{c}w \\ j\end{array}\right), w, j=1,2,3$ in (45) and (46) is the binomial coefficient.

Finally, the next vectorial parameterization is obtained from (44):

$$
\chi(t)=\Phi_{K}(t) \Theta_{K}+I_{5}[r(t)]+I_{5}[\lambda(t)]
$$

with $\chi(t) \in R^{3 n \times 1}, \Phi_{K}(t) \in R^{3 n \times(17 n-10)}$ is the regressor, and $\Theta_{K} \in R^{(17 n-10) \times 1}$ is the vector containing the stiffness/mass ratios that will be estimated.

Proposition 4. The term $I_{5}[r(t)]$ in (47) converges to zero at $t=5 \delta$.

Proof. See Appendix B.

Moreover, by substituting the variable $\Lambda(s)$ (40) into (43) allows obtaining the Laplace transform of $\mathscr{L}\left(I_{5}[\lambda]\right)$, which is given by

$$
\begin{aligned}
\mathscr{L}\left(I_{5}[\lambda]\right)= & {\left[\Sigma(s)+\Sigma_{g}(s)\right] H_{3}(s) } \\
& +\left(a_{0} \Sigma(s)-a_{1} \Upsilon(s) \Theta_{K}\right) H_{2}(s) \\
& -\Upsilon(s) \Theta_{K} H_{1}(s), \\
H_{1}(s)= & s \delta G(s), \\
H_{2}(s)= & s^{2} \delta G(s), \\
H_{3}(s)= & s^{3} \delta G(s),
\end{aligned}
$$

where $G(s)$ is the following fifth-order low pass filter:

$$
G(s)=\left(\frac{1-e^{-\delta s}}{\delta s}\right)^{5}
$$

Its frequency response is given by

$$
\begin{aligned}
|G(j \omega)| & =\left|\frac{\sin \left(\pi \omega / \omega_{c}\right)}{\left(\pi \omega / \omega_{c}\right)}\right|^{5}, \\
\omega_{c} & =\frac{2 \pi}{\delta}, \\
f_{c} & =\frac{1}{\delta},
\end{aligned}
$$

where $\omega_{c}$ and $f_{c}$ determine the bandwidth of $G(s)$ and are given in $\mathrm{rad} / \mathrm{s}$ and $\mathrm{Hz}$, respectively.

Let $\omega_{1}, \omega_{2}, \ldots, \omega_{3 n}=\omega_{\max }$ be all the natural frequencies of the structure, which are ordered from the lowest to the highest frequency. If the structure is completely instrumented, it is 
suggested that the frequency $\omega_{c}$ in (51) is selected such that it takes a value within the interval $\omega_{c} \in\left[2 \omega_{\max }, 4 \omega_{\max }\right]$. Values of $\omega_{c}$ above this interval could deteriorate the performance of the identification method, since high frequency noise could be passed by the filters in (49). On the other hand, if only $n_{r}$ floors are instrumented, where $n_{r}<n$ (see Remark 2), it is recommended to select $\delta$ such that $\omega_{c}$ takes the value within $\omega_{c} \in\left[1.5 \omega_{3 * n_{r}}, 2 \omega_{3 * n_{r}}\right]$; for example, if a five-story structure is instrumented in only four floors, then $\delta$ would be chosen such that $\omega_{c} \in\left[1.5 \omega_{12}, 2 \omega_{12}\right]$. Note that, in the reduced measurement case, only the first $3 n_{r}$ natural frequencies of the torsional building will be estimated, since a $n_{r}$-story building model will be obtained.

\section{Parameter Identification of the Building}

4.1. Estimation of the Stiffness/Mass Ratios. The expression (47) is valid for $t=k T_{s}, k=0,1,2, \ldots$, where $T_{s}$ is the sampling period. Then, (47) can be written as

$$
\chi\left(k T_{s}\right)=\Phi_{K}\left(k T_{s}\right) \Theta_{K}+I_{5}\left[\lambda\left(k T_{s}\right)\right], \quad t \geq 5 \delta .
$$

Omitting $T_{s}$ leads to

$$
\chi(k)=\Phi_{K}(k) \Theta_{K}+I_{5}[\lambda(k)] .
$$

In order to estimate the stiffness/mass ratios of the structure, the vector parameterization is combined with the Least Squares Method (LSM) [26], which is given by

$$
\widehat{\Theta}_{K}=\left[\sum_{k=1}^{N} \Phi_{K}^{T}(k) \Phi_{K}(k)\right]^{-1} \sum_{k=1}^{N} \Phi_{K}^{T}(k) \chi(k),
$$

where $N$ is the total number of samples of $\Phi_{K}$ and $\chi$.

The stiffness/mass ratios can be also estimated online by means of the recursive LSM, given by

$$
\begin{aligned}
& \widehat{\Theta}_{K}(k)=\widehat{\Theta}_{K}(k-1)+L(k) \epsilon(k), \\
& L(k)=P(k-1) \Phi_{K}^{T}(k) \\
& \cdot\left[\beta I_{3 n \times 3 n}+\Phi_{K}(k) P(k-1) \Phi_{K}^{T}(k)\right]^{-1}, \\
& P(k)=\frac{\left[P(k-1)-L(k) \Phi_{K}(k) P(k-1)\right]}{\beta}, \\
& \epsilon(k)=\chi(k)-\Phi_{K}(k) \widehat{\Theta}_{K},
\end{aligned}
$$

where $\epsilon(k)$ is the output estimation error, $\beta, 0<\beta \leq 1$, is the forgetting factor, and $P=P^{T} \in R^{17 n-10 \times 17 n-10}$ is the covariance matrix such that $P>0$. Note that the online LSM allows detecting damage in a seismically excited structure by observing the variations of the parameter estimates.

It is worth mentioning that, according to Proposition 4, taking samples of $\chi$ and $\Phi_{K}$ from $t=5 \delta$ to a final time $t_{f}$ assures that the LSM is insensible to constant disturbances.

4.2. Estimation of the Modal Parameters. Once that vector $\widehat{\Theta}_{K}$ has been estimated, it is possible to construct matrices
$\widehat{\mathbb{M}^{-1} \mathbb{K}}$ and $\widehat{\mathbb{M}^{-1} \mathbb{C}}$ of the building model (23). Matrix $\widehat{\mathbb{M}^{-1} \mathbb{C}}$ is obtained through the next expression:

$$
\widehat{\mathbb{M}^{-1} \mathbb{C}}=a_{0}+a_{1} \widehat{\mathbb{M}^{-1} \mathbb{K}}
$$

The natural frequencies and modal damping of the estimated building model can be obtained by means of the roots of the next characteristic polynomial corresponding to model (23):

$$
I_{3 n \times 3 n} s^{2}+\widehat{\mathbb{M}^{-1} \mathbb{C}} s+\widehat{\mathbb{M}^{-1} \mathbb{K}}=O_{3 n \times 3 n} .
$$

The roots of polynomial (57) are given by

$$
\begin{aligned}
& \gamma_{i}=-\widehat{\alpha}_{i}+j \widehat{\omega}_{d i}, \\
& \gamma_{i}^{*}=-\widehat{\alpha}_{i}-j \widehat{\omega}_{d i}, \\
& \qquad i=1,2, \ldots, 3 n \\
& \widehat{\alpha}_{i}=\widehat{\zeta}_{i} \widehat{\omega}_{i}, \\
& \widehat{\omega}_{d i}=\widehat{\omega}_{i} \sqrt{1-\widehat{\zeta}_{i}^{2}},
\end{aligned}
$$

where $\widehat{\omega}_{i}$ and $\widehat{\zeta}_{i}, i=1,2, \ldots, 3 n$, are the estimated natural frequencies and damping ratios, respectively. They are computed as

$$
\begin{aligned}
& \widehat{\omega}_{i}=\sqrt{\widehat{\alpha}_{i}^{2}+\widehat{\omega}_{d i}^{2}}, \\
& \widehat{\zeta}_{i}=\frac{\widehat{\alpha}_{i}}{\widehat{\omega}_{i}}
\end{aligned}
$$

$$
i=1,2, \ldots, 3 n \text {. }
$$

It is worth mentioning that the estimated modal shape matrix can be obtained by computing the eigenvectors of the matrix $\widehat{\mathbb{M}^{-1} \mathbb{K}}$.

4.3. Estimation of the Eccentricities and Radii of Gyration. In order to identify the eccentricities $e_{x 1,1}, e_{x 1,2}, \ldots, e_{x n, n}$ of the structure, assume that the estimates $\widehat{\theta}_{K y \theta z}$ of $\theta_{K y \theta z}$ in (A.5), $z=1,2, \ldots, 3 n-3$, satisfy the following equalities:

$$
\begin{aligned}
\widehat{\theta}_{K y \theta(3 i)} & =\left(\frac{e_{x i, i+1} K_{y i+1}}{m_{i+1}}\right)=\widehat{e}_{x i, i+1} \frac{\widehat{K_{y i+1}}}{m_{i+1}} \\
& =\widehat{e}_{x i, i+1} \widehat{\theta}_{K y y(2 i+1)}, \\
\widehat{\theta}_{K y \theta(3 i-1)} & =\left(\frac{e_{x i+1, i+1} K_{y i+1}}{m_{i}}\right)=\widehat{e}_{x i+1, i+1} \frac{\widehat{K_{y i+1}}}{m_{i}} \\
& =\widehat{e}_{x i+1, i+1} \widehat{\theta}_{K y y(2 i)}, \\
\widehat{\theta}_{K y \theta 1} & =\left(\frac{e_{x 1,1} K_{y 1}}{m_{1}}+\frac{e_{x 1,2} K_{y 2}}{m_{1}}\right)
\end{aligned}
$$




$$
\begin{aligned}
& =\widehat{e}_{x 1,1} \frac{\widehat{K_{y 1}}}{m_{1}}+\widehat{e}_{x 1,2} \frac{\widehat{K_{y 2}}}{m_{1}} \\
& =\widehat{e}_{x 1,1} \widehat{\theta}_{K y y 1}+\widehat{e}_{x 1,2} \widehat{\theta}_{K y y 2},
\end{aligned}
$$

where $i=1,2, \ldots, n-1$. From (60), the next estimates $\widehat{e}_{x 1,1}, \ldots, \widehat{e}_{x n, n}$ of the eccentricities $e_{x 1,1}, \ldots, e_{x n, n}$ are given by

$$
\begin{aligned}
\hat{e}_{x i, i+1} & =\frac{\widehat{\theta}_{K y \theta(3 i)}}{\widehat{\theta}_{K y y(2 i+1)}}, \\
\hat{e}_{x i+1, i+1} & =\frac{\widehat{\theta}_{K y \theta(3 i-1)}}{\widehat{\theta}_{K y y(2 i)}}, \\
\widehat{e}_{x 1,1} & =\frac{\widehat{\theta}_{K y \theta 1}-\widehat{e}_{x 1,2} \widehat{\theta}_{K y y 2}}{\widehat{\theta}_{K y y 1}} .
\end{aligned}
$$

A similar procedure allows obtaining the estimates $\widehat{e}_{y 1,1}, \ldots$, $\widehat{e}_{y n, n}$ of the eccentricities $e_{y 1,1}, \ldots, e_{y n, n}$.

On the other hand, suppose that the estimates $\widehat{\theta}_{K y \theta j \star}$ of $\theta_{K y \theta j \star}$ in (A.7), $j=1,2, \ldots, n$, fulfill the next equality:

$$
\begin{aligned}
\widehat{\theta}_{K y \theta(2 j-1) \star} & =\left(\frac{e_{x j, j} K_{y j}}{m_{j} r_{j}^{2}}\right)=\frac{1}{\widehat{r}_{j}^{2}} \widehat{e}_{x j, j} \frac{\widehat{K_{y j}}}{m_{j}} \\
& =\frac{1}{\widehat{r}_{j}^{2}} \widehat{e}_{x j, j} \widehat{\theta}_{K y y(2 j-1)}, \quad j=1,2, \ldots, n .
\end{aligned}
$$

Then, the radius of gyration can be estimated as

$$
\widehat{r}_{j}=\sqrt{\frac{\widehat{e}_{x j, j} \widehat{\theta}_{K y y(2 j-1)}}{\widehat{\theta}_{K y \theta(2 j-1) \star}}}, \quad j=1,2, \ldots, n .
$$

4.4. Estimation of the Mass, Stiffness, and Damping Matrices. The next proposed methodology uses the knowledge of a building floor mass in order to identify the mass, stiffness, and damping matrices of the structure. Employing the entries of the estimated vectors $\widehat{\theta}_{K x x}, \widehat{\theta}_{K y y}$, and $\widehat{\theta}_{K \theta \theta}$ of $\theta_{K x x}, \theta_{K y y}$, and $\theta_{K \theta \theta}$ given in (A.1) and assuming the knowledge of the mass $m_{1}$, it is possible to compute the entries of the mass and stiffness matrices as follows:

$$
\begin{aligned}
\widehat{K}_{x 1} & =m_{1} \widehat{\theta}_{K x x 1}, \\
\widehat{K}_{x 2} & =m_{1} \widehat{\theta}_{K x x 2}, \\
\widehat{m}_{2} & =\frac{\widehat{K}_{x 2}}{\widehat{\theta}_{K x x 3}}, \\
\widehat{K}_{x 3} & =\widehat{m}_{2} \widehat{\theta}_{K x x 4}, \\
\widehat{m}_{3} & =\frac{\widehat{K}_{x 3}}{\widehat{\theta}_{K x x 5}}, \\
\widehat{K}_{x s} & =\widehat{m}_{s-1} \widehat{\theta}_{K x x(2 s-2)},
\end{aligned}
$$

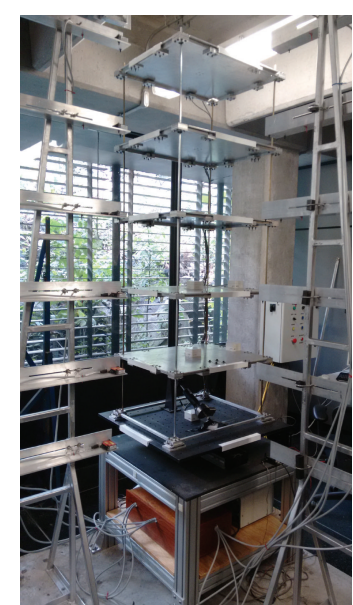

Figure 2: Torsionally coupled five-story building mounted on the shaking table.

$$
\begin{aligned}
\widehat{m}_{s} & =\frac{\widehat{K}_{x s}}{\widehat{\theta}_{K x x(2 s-1)},} \\
\widehat{K}_{y 1} & =m_{1} \widehat{\theta}_{K y y 1}, \\
\widehat{K}_{y 2} & =m_{1} \widehat{\theta}_{K y y 2}, \\
\widehat{K}_{y s} & =\widehat{m}_{s-1} \widehat{\theta}_{K y y(2 s-2)}, \\
\widehat{K}_{9(2 i-1)} & =\widehat{m}_{i} \widehat{r}_{i}^{2} \widehat{\theta}_{K \theta \theta(3 i-2)}, \\
\widehat{K}_{9(2 j)} & =\widehat{m}_{j} \widehat{r}_{j}^{2} \widehat{\theta}_{K \theta \theta(3 j-1)},
\end{aligned}
$$

where $s=2,3, \ldots, n, i=1,2, \ldots, n$, and $j=1,2, \ldots, n-1$. The terms $\widehat{r}_{1}^{2}, \widehat{r}_{2}^{2}, \ldots, \widehat{r}_{n}^{2}$ are given in (63); on the other hand, an estimate $\widehat{\mathbb{C}}$ of the damping matrix $\mathbb{C}$ can be computed by the next expression:

$$
\widehat{\mathbb{C}}=\widehat{\mathbb{M}} \widehat{\mathbb{M}^{-1} \mathbb{C}}
$$

where the entries of the matrix $\widehat{\mathbb{M}}$ are the parameters $m_{1}, \widehat{m}_{2}$, $\ldots, \widehat{m}_{n}$ given in (64); moreover, $\widehat{\mathbb{M}^{-1} \mathbb{C}}$ is defined in (56). The procedure is similar if any other mass $m_{i}$ is assumed to be known.

Remark 5. Note that a parameter projection scheme as the one presented in [19] can be applied to the estimates of the denominators in (61), (63), and (64) in order to avoid divisions by zero and to recursively identify the eccentricities, radii of gyration, and the stiffness, mass, and damping matrices.

\section{Experimental Results}

An experimental five-story torsional building, which is shown in Figure 2, is used to verify the performance of the vector parameterization combined with the LSM. The signals $\chi(45)$ and $\Phi_{K}$ (46) of the parameterization and the LSM are 


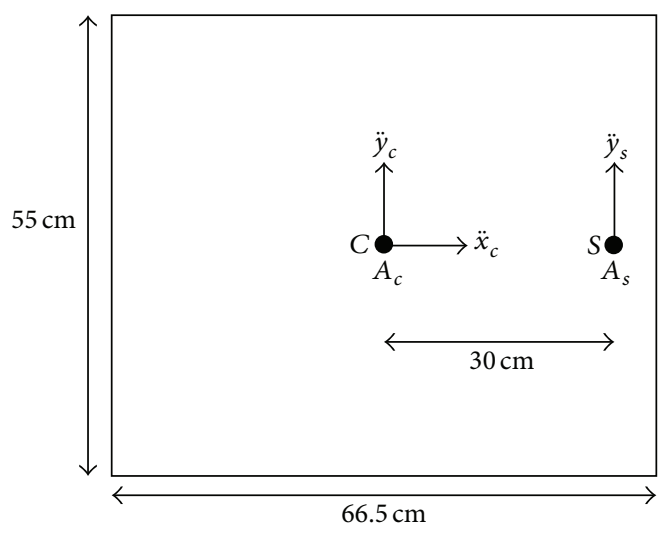

(a) Position of the accelerometers

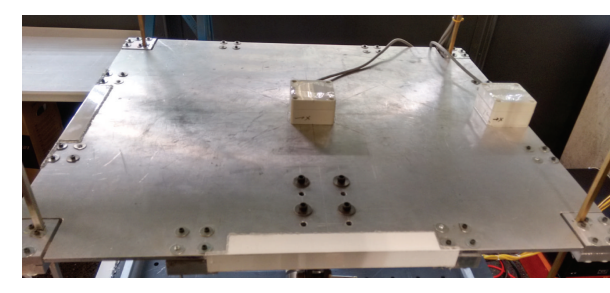

(b) Photo of the accelerometers

Figure 3: Position of the accelerometers in the floors.

coded using the Matlab/Simulink software. All the experiments use a sampling time $T_{s}$ of $2 \mathrm{~ms}$ and the integrals of $\Phi_{K}$ and $\chi$ are computed through the trapezoidal numerical integration method. The experimental structure has dimensions $65 \times 55 \times 175.5 \mathrm{~cm}$ and is mounted on a shaking table, which is actuated by servomotors from Parker model 406T03LXR, that moved it over the $x$-and $y$-axes of the horizontal plane. Each floor is made from aluminum; moreover, the center of mass and resistance of each story do not coincide since only one column is made from aluminum and the remaining three columns are made from brass. Note that the stiffness of a brass column is larger that the one of an aluminum column. The dimensions of the columns of the first and the remaining four floors are $0.635 \times 0.635 \times 31.5 \mathrm{~cm}$ and $0.635 \times 0.635 \times$ $36 \mathrm{~cm}$, respectively; in addition, the masses of the floors are approximately given by $m_{1}=10.78 \mathrm{~kg}$ and $m_{2}=m_{3}=m_{4}=$ $m_{5}=9.2 \mathrm{~kg}$. It is important to mention that the first floor is heavier that the others, since an iron mass of approximately $1.58 \mathrm{~kg}$ is added to its edge. Twelve laser sensors from MicroEpsilon, model optoNCDT 1302, measure the absolute position of the shaking table and floors; these sensors are not used for parameter identification purposes. Two PCI-6221 boards from National Instruments perform the data acquisition and their communication with a personal computer is carried out using the Matlab Real-Time Windows Target toolbox. The absolute accelerations of the shaking table and floors are measured through dual-axis accelerometers from Analog Devices, model ADXL203, that have a range from $-1.7 \mathrm{~g}$ to $1.7 \mathrm{~g}$ and can provide accelerations in the $x$ - and $y$-axes. The center of the shaking table contains an accelerometer that measures its acceleration in both axes; moreover, each floor is equipped with two accelerometers, represented as $A_{c}$ and $A_{s}$, as shown in Figure 3 ; accelerometer $A_{c}$ provides the absolute accelerations of the center of mass (CM) of the floor in the $x$ and $y$ directions, which are denoted as $\ddot{x}_{c}$ and $\ddot{y}_{c}$; on the other side, accelerometer $A_{s}$ provides the absolute acceleration at the point $S$ in the $y$ direction; this acceleration is denoted as $\ddot{y}_{s}$. In order to compute the angular acceleration $\ddot{\theta}_{i m}$ of the $i$ th floor, the following approximation is employed [13]:

$$
\ddot{\theta}_{i m} \approx \frac{\ddot{y}_{i s}-\ddot{y}_{i c}}{0.3 \mathrm{~m}}, \quad i=1,2,3,4,5,
$$

TABLE 1: Natural frequencies of the structure.

\begin{tabular}{lc}
\hline $\mathrm{rad} / \mathrm{s}$ & $\mathrm{Hz}$ \\
\hline$\omega_{1}=9.588$ & $f_{1}=1.526$ \\
$\omega_{2}=9.971$ & $f_{2}=1.587$ \\
$\omega_{3}=14.476$ & $f_{3}=2.304$ \\
$\omega_{4}=29.814$ & $f_{4}=4.745$ \\
$\omega_{5}=30.775$ & $f_{5}=4.898$ \\
$\omega_{6}=43.335$ & $f_{6}=6.897$ \\
$\omega_{7}=46.213$ & $f_{7}=7.355$ \\
$\omega_{8}=48.418$ & $f_{8}=7.706$ \\
$\omega_{9}=60.978$ & $f_{9}=9.705$ \\
$\omega_{10}=63.020$ & $f_{10}=10.030$ \\
$\omega_{11}=68.550$ & $f_{11}=10.910$ \\
$\omega_{12}=72.005$ & $f_{12}=11.460$ \\
$\omega_{13}=72.319$ & $f_{13}=11.510$ \\
$\omega_{14}=90.289$ & $f_{14}=14.370$ \\
$\omega_{15}=110.081$ & $f_{15}=17.520$ \\
\hline
\end{tabular}

where $\ddot{y}_{i c}$ and $\ddot{y}_{i s}$ are the absolute accelerations of the $i$ th floor at the points $C$ and $S$, respectively, and $0.3 \mathrm{~m}$ is the distance between the accelerometers in the $x$ direction, as depicted in Figure 3(a). On the other hand, the relative accelerations $\ddot{x}_{i m}$ and $\ddot{y}_{i m}$ of the $i$ th floor, which are employed in the matrices $\ddot{U}_{m}$ and $\ddot{W}_{m}$ in (45)-(46), are obtained as follows:

$$
\begin{aligned}
& \ddot{x}_{i m}=\ddot{x}_{i c}-\ddot{u}_{g x m}, \\
& \ddot{y}_{i m}=\ddot{y}_{i c}-\ddot{u}_{g y m}, \\
& \quad i=1,2,3,4,5,
\end{aligned}
$$

where $\ddot{u}_{g x m}$ and $\ddot{u}_{g y m}$ are the accelerations provided by the accelerometer of the shaking table.

Let $\omega_{i}$ and $f_{i}, i=1,2, \ldots, 15$, be the natural frequencies of the building in rad/s and $\mathrm{Hz}$, respectively. Table 1 presents the natural frequencies of the structure that are obtained by exciting it through a chirp signal from 0.1 to $30 \mathrm{~Hz}$. The natural frequencies are between 9.588 and $110.081 \mathrm{rad} / \mathrm{s}$ or 1.526 and $17.520 \mathrm{~Hz}$, and they are also shown in Figure 4, 


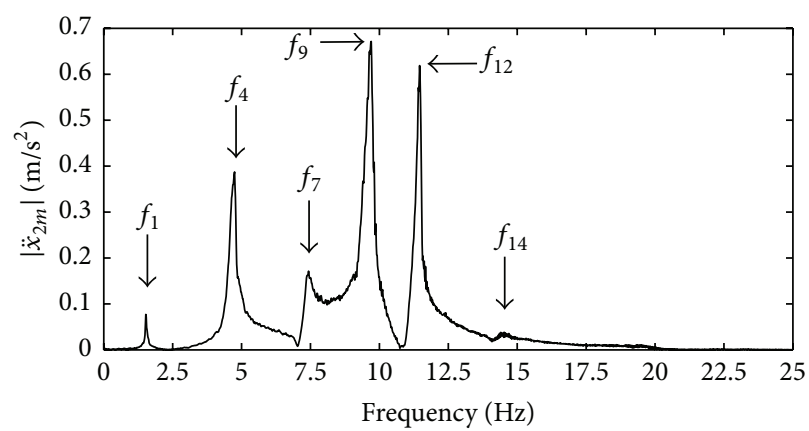

(a) Fourier spectra of $\ddot{x}_{2 m}$

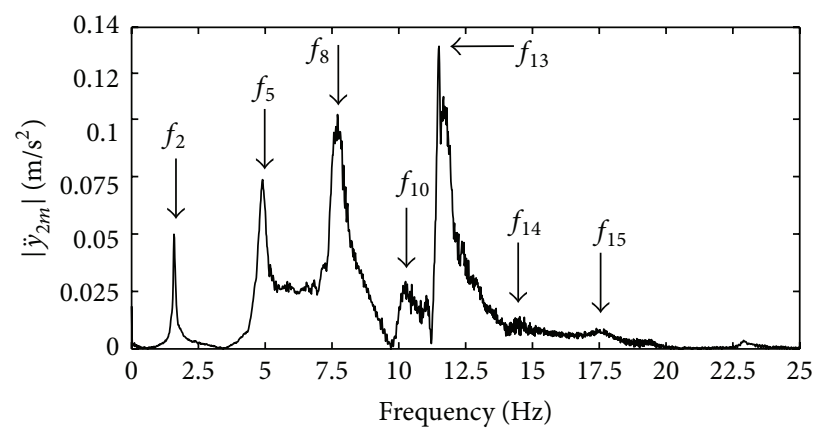

(b) Fourier spectra of $\ddot{y}_{2 m}$

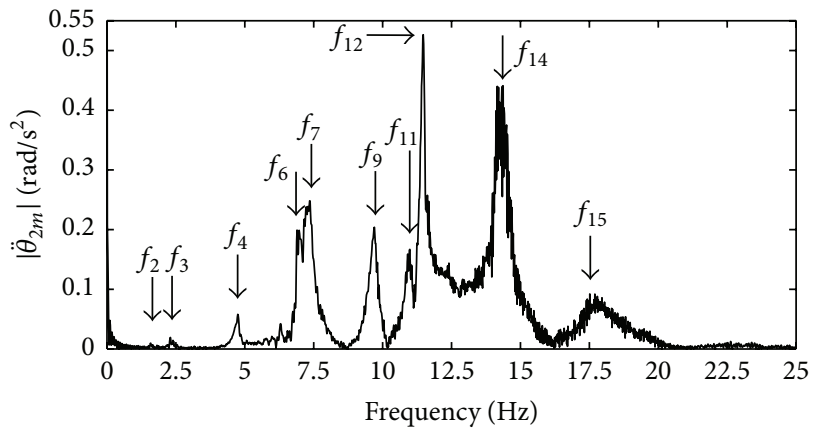

(c) Fourier spectra of $\ddot{\theta}_{2 m}$

FIGURE 4: Fourier spectra of the acceleration measurements $\ddot{x}_{2 m}, \ddot{y}_{2 m}$, and $\ddot{\theta}_{2 m}$ of the second floor.

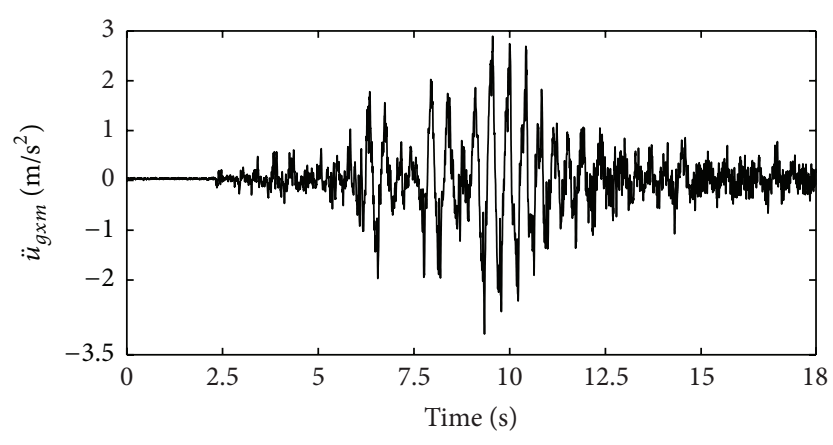

(a) East-West acceleration $\ddot{u}_{g x m}$

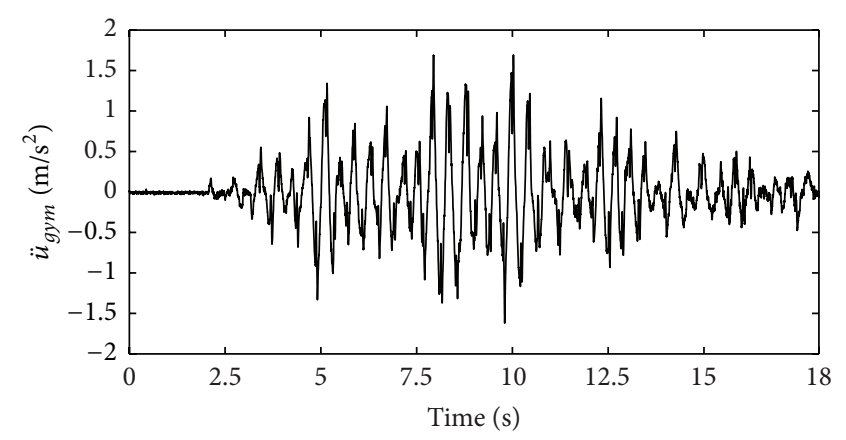

(b) North-South acceleration $\ddot{u}_{\text {gym }}$

FIGURE 5: Acceleration measurements $\ddot{u}_{g x m}$ and $\ddot{u}_{\text {gym }}$ of the shaking table.

which depicts the Fourier spectra of the acceleration measurements $\ddot{x}_{2 m}, \ddot{y}_{2 m}$, and $\ddot{\theta}_{2 m}$ of the second floor. This chirp signal is not used for estimating the parameter vector $\Theta_{K}$ of the proposed parameterization given in (53). In order to estimate $\Theta_{K}$, the experimental structure is excited through the North-South and East-West components of the Mexico City 1985 earthquake, which is fitted in amplitude to be in agreement with the structure. Figures 5 and 6 depict the acceleration measurements of the shaking table and fourth floor, respectively; note that these measurements have offsets. Notice in Figure 4 that the lower frequencies in Table 1 have a small contribution in the spectra, a fact that will have an impact on the identification of the lower frequencies as it will be shown later.

\subsection{Experimental Results Using the Acceleration Measurements} of All the Floors. This section presents the identified model of the structure obtained using acceleration measurements of all the floors and ground. It is assumed that estimates $\widehat{\zeta}_{1 \star}$ and $\widehat{\zeta}_{2 \star}$ of the damping ratios $\zeta_{1}$ and $\zeta_{2}$ of the structure are equal to $\widehat{\zeta}_{1 \star}=\widehat{\zeta}_{2 \star}=1 \%$. This assumption that the first and second modes of the structure are equal is reasonable based on experimental data [17]. The parameters $\widehat{\zeta}_{1 \star}$ and $\widehat{\zeta}_{2 \star}$ 


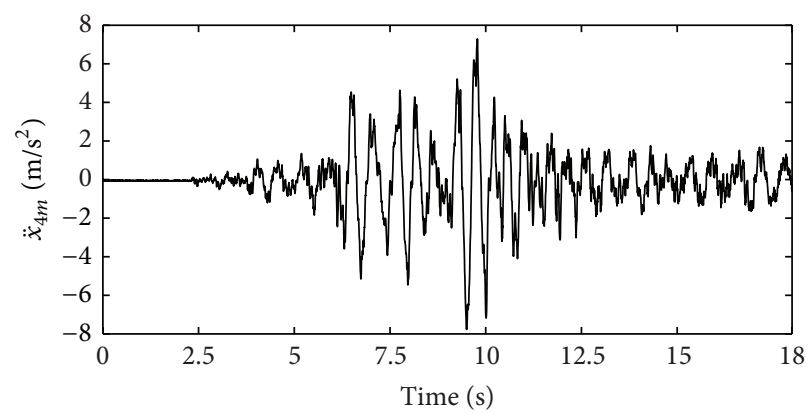

(a) Measured acceleration $\ddot{x}_{4 m}$

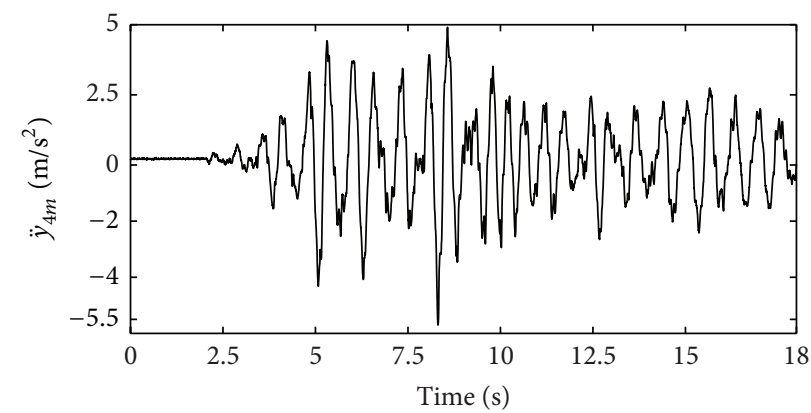

(b) Measured acceleration $\ddot{y}_{4 m}$

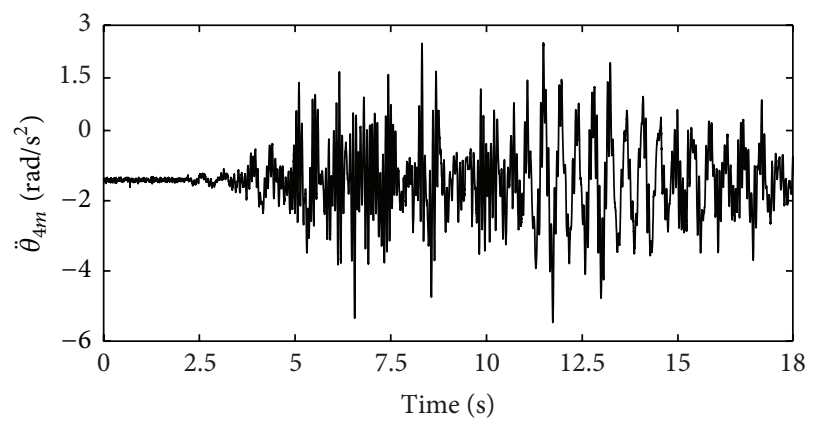

(c) Measured acceleration $\ddot{\theta}_{4 m}$

Figure 6: Measured accelerations $\ddot{x}_{4 m}, \ddot{y}_{4 m}$, and $\ddot{\theta}_{4 m}$ of the fourth floor that are produced by the Mexico City 1985 earthquake.

and the natural frequencies $\widehat{\omega}_{1 \star}=\omega_{1}$ and $\widehat{\omega}_{2 \star}=\omega_{2}$ are substituted into (16) in order to compute the constants $a_{0}$ and $a_{1}$, corresponding to the Rayleigh damping (14), whose values are given by

$$
\begin{aligned}
& a_{0}=0.09776, \\
& a_{1}=0.001022 .
\end{aligned}
$$

Note that these constants are employed in the signals $\chi(45)$ and $\Phi_{K}$ in (46) of the proposed vector parameterization.

Tables 2 and 3 show, respectively, the identified natural frequencies and damping ratios obtained for three different values of the integration time period $\delta$, which are $\delta_{1}=$ $0.016 \mathrm{~s}, \delta_{2}=0.02 \mathrm{~s}$, and $\delta_{3}=0.024 \mathrm{~s}$. These modal parameters are computed using (59) and matrices $\widehat{\mathbb{M}^{-1} \mathbb{C}}$ and $\widehat{\mathbb{M}^{-1} \mathbb{K}}$ in (57), which are constructed using the vector estimate $\widehat{\Theta}_{K}$ produced by the offline LSM (54), which takes samples of $\chi$ and $\Phi_{K}$ from $5 \delta$ to $18 \mathrm{~s}$. Table 2 also shows the identification error $\widetilde{\omega}_{i}$ in percentage (\%) for the estimated natural frequency $\omega_{i}$, which is defined as

$$
\widetilde{\omega}_{i}=\frac{\left|\widehat{\omega}_{i}-\omega_{i}\right|}{\omega_{i}} \times 100, \quad i=1,2, \ldots, n,
$$

where $n=15$; note that the natural frequencies are identified with an error less than $15 \%$ and that the larger errors in Table 2 correspond to frequencies with low magnitude in Figure 4. On the other hand, the following index $E_{\widetilde{\omega}}$,

$$
E_{\widetilde{\omega}}=\sqrt{\sum_{i=1}^{n} \widetilde{\omega}_{i}^{2}}
$$

is also presented in Table 2 and it is computed in order to validate the quality of the identified model; the smaller the value of $E_{\widetilde{\omega}}$, the better the quality of the identified building model. Moreover, Figures 8(a) and 8(b) exhibit the graphs $E_{\widetilde{\omega}}$ versus $\delta$ and $E_{\widetilde{\omega}}$ versus $\omega_{c}$, respectively. Note that relative low values of $E_{\widetilde{\omega}}$ appear within $\delta=[0.014,0.028]$ s or within $\omega_{c}=\left[2 \omega_{15}, 4 \omega_{15}\right] \mathrm{rad} / \mathrm{s}$.

Let $\widehat{\ddot{v}}_{i}, v=x, y, \theta, i=1,2, \ldots, n$, be the responses predicted through the identified model. Figure 7 shows the timehistory responses $\widehat{\ddot{x}}_{4}$ and $\widehat{\ddot{y}}_{5}$, corresponding to the identified model obtained with $\delta=\delta_{1}$, and they are compared with the acceleration measurements $\ddot{x}_{4 m}$ and $\ddot{y}_{5 m}$, respectively. It is shown that these estimated responses are close to the measured ones.

Table 4 shows the nominal and estimated eccentricities and radii of gyration of the building; these estimates are produced by (61) and (63) and the stiffness/mass ratios of the vector $\widehat{\Theta}_{K}$ identified with the offline LSM with $\delta=\delta_{1}$. From Table 4 , it is possible to see that the estimated radii of gyration are between 23.38 and $27.76 \mathrm{~cm}$; moreover, the estimated eccentricities in the $x$ - and $y$-axes take a value between 1.24 and $3.92 \mathrm{~cm}$ and -0.26 and $10.66 \mathrm{~cm}$, respectively. It is important to mention that $e_{y 1,1}$ has a high value since the iron mass added to the edge of the first floor moves the center of mass of this story away from its geometric center.

To compare the obtained results and to analyze the larger errors in the lower frequencies, the nominal values of the experimental structure were computed by measuring the mass of each floor and calculating the stiffness using the dimensions, moments of inertia, and the columns' Young 
TABLE 2: Estimated natural frequencies.

\begin{tabular}{lcccccc}
\hline Natural frequency $(\mathrm{rad} / \mathrm{s})$ & Estimate $\widehat{\omega}_{i}$ with $\delta_{1}$ & Error $(\%)$ & Estimate $\widehat{\omega}_{i}$ with $\delta_{2}$ & Error $(\%)$ & Estimate $\widehat{\omega}_{i}$ with $\delta_{3}$ & Error $(\%)$ \\
\hline$\omega_{1}=9.588$ & 10.948 & $\widetilde{\omega}_{1}=14.186$ & 10.785 & $\widetilde{\omega}_{1}=12.485$ & 10.752 & $\widetilde{\omega}_{1}=12.139$ \\
$\omega_{2}=9.971$ & 11.372 & $\widetilde{\omega}_{2}=14.048$ & 11.244 & $\widetilde{\omega}_{2}=12.763$ & 11.153 & $\widetilde{\omega}_{2}=11.853$ \\
$\omega_{3}=14.476$ & 14.696 & $\widetilde{\omega}_{3}=1.518$ & 14.005 & $\widetilde{\omega}_{3}=3.2540$ & 14.572 & $\widetilde{\omega}_{3}=0.659$ \\
$\omega_{4}=29.814$ & 31.937 & $\widetilde{\omega}_{4}=7.120$ & 31.831 & $\widetilde{\omega}_{4}=6.765$ & 31.766 & $\widetilde{\omega}_{4}=6.548$ \\
$\omega_{5}=30.775$ & 32.834 & $\widetilde{\omega}_{5}=6.690$ & 32.560 & $\widetilde{\omega}_{5}=5.799$ & 32.346 & $\widetilde{\omega}_{5}=5.103$ \\
$\omega_{6}=43.335$ & 45.563 & $\widetilde{\omega}_{6}=5.141$ & 45.015 & $\widetilde{\omega}_{6}=3.877$ & 44.636 & $\widetilde{\omega}_{6}=3.003$ \\
$\omega_{7}=46.213$ & 48.677 & $\widetilde{\omega}_{7}=5.332$ & 48.498 & $\widetilde{\omega}_{7}=4.944$ & 48.368 & $\widetilde{\omega}_{7}=4.664$ \\
$\omega_{8}=48.418$ & 50.344 & $\widetilde{\omega}_{8}=3.978$ & 49.978 & $\widetilde{\omega}_{8}=3.221$ & 49.678 & $\widetilde{\omega}_{8}=2.603$ \\
$\omega_{9}=60.978$ & 62.049 & $\widetilde{\omega}_{9}=1.756$ & 61.882 & $\widetilde{\omega}_{9}=1.482$ & 61.729 & $\widetilde{\omega}_{9}=1.231$ \\
$\omega_{10}=63.020$ & 63.904 & $\widetilde{\omega}_{10}=1.402$ & 63.524 & $\widetilde{\omega}_{10}=0.800$ & 63.196 & $\widetilde{\omega}_{10}=0.280$ \\
$\omega_{11}=68.550$ & 69.844 & $\widetilde{\omega}_{11}=1.889$ & 69.389 & $\widetilde{\omega}_{11}=1.224$ & 68.910 & $\widetilde{\omega}_{11}=0.5255$ \\
$\omega_{12}=72.005$ & 71.580 & $\widetilde{\omega}_{12}=0.591$ & 71.275 & $\widetilde{\omega}_{12}=1.014$ & 71.015 & $\widetilde{\omega}_{12}=1.375$ \\
$\omega_{13}=72.319$ & 72.729 & $\widetilde{\omega}_{13}=0.567$ & 72.243 & $\widetilde{\omega}_{13}=0.106$ & 71.791 & $\widetilde{\omega}_{13}=0.731$ \\
$\omega_{14}=90.289$ & 89.871 & $\widetilde{\omega}_{14}=0.463$ & 89.087 & $\widetilde{\omega}_{14}=1.332$ & 88.279 & $\widetilde{\omega}_{14}=2.226$ \\
$\omega_{15}=110.081$ & 100.990 & $\widetilde{\omega}_{15}=8.258$ & 100.112 & $\widetilde{\omega}_{15}=9.056$ & 99.030 & $\widetilde{\omega}_{15}=10.040$ \\
& & $E_{\widetilde{\omega}}=25.392$ & & $E_{\widetilde{\omega}}=23.405$ & & $E_{\widetilde{\omega}}=22.468$ \\
\hline
\end{tabular}

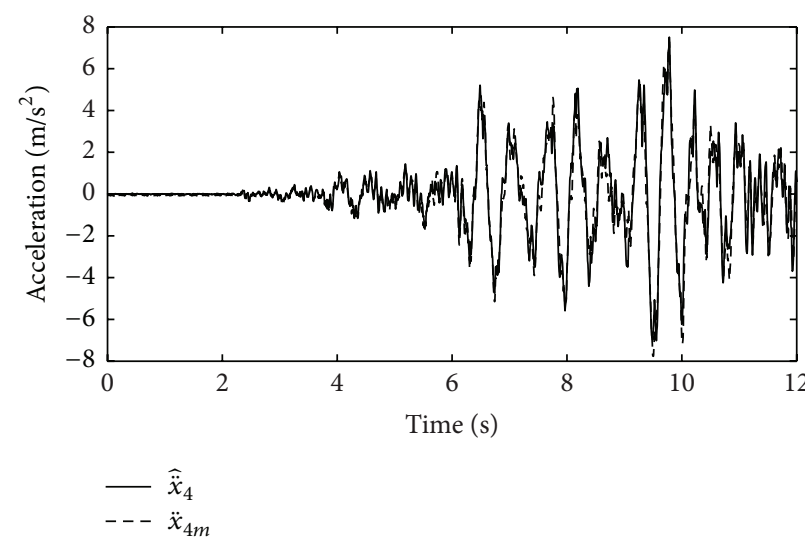

(a) Comparison between $\widehat{\ddot{x}}_{4}$ and $\ddot{x}_{4 m}$

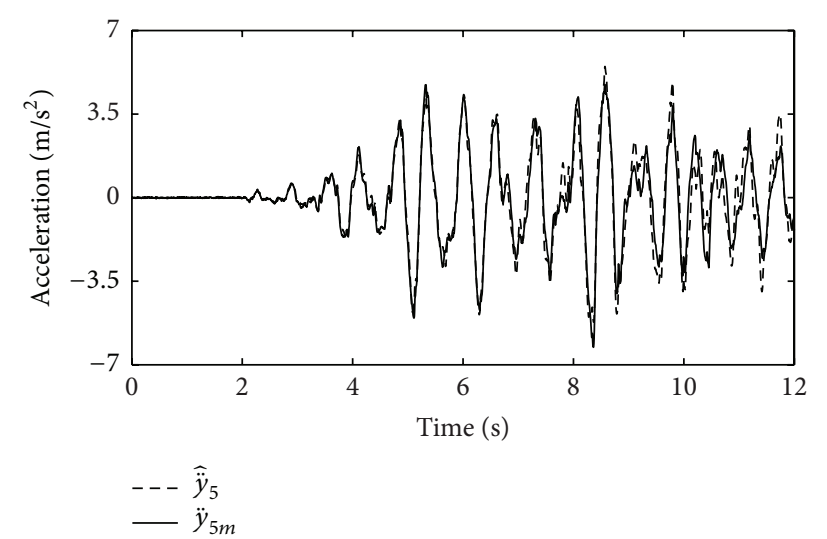

(b) Comparison between $\widehat{\ddot{y}}_{5}$ and $\ddot{y}_{5 m}$

FiguRE 7: Comparison between predicted responses by the identified model and the acceleration measurements.

elastic modulus of the experimental building [17]. Using the precise knowledge of the mass of the first floor $m_{1}=$ $10.78 \mathrm{~kg}$ and the final estimates of the vector of parameters, the estimated mass and stiffness matrices were computed according to the procedure detailed in Section 4. The nominal natural frequencies are the ones obtained by substituting the nominal mass and stiffness matrices into (5) and (7). The results are presented in Table 5 that shows that most of the entries of the estimated mass and stiffness matrices and most of the natural frequencies are close to their nominal values. Also note that the first two estimated natural frequencies $\widehat{\omega}_{1}$ and $\widehat{\omega}_{2}$ are, respectively, closer to the frequencies $\omega_{1}^{\text {nom }}$ and $\omega_{2}^{\text {nom }}$ than to the frequencies $\omega_{1}$ and $\omega_{2}$ in Table 1.

Remark 6. The estimated natural frequencies $\widehat{\omega}_{i}, i=1,2$, $\ldots, n$, can also be calculated using the nominal frequencies $\omega_{i}^{\text {nom }}$ in Table 5 instead of the frequencies $\omega_{i}$ in Table 1 , which are obtained with the Fourier spectra of the acceleration measurements. In other words, the index $E_{\widetilde{\omega}}$ in (70) could have been calculated using $\omega_{i}^{\text {nom }}$ in place of $\omega_{i}$. Since the natural frequencies, computed with the Fourier spectra, are usually selected in the literature for model validation $[8,13]$, this paper employs the frequencies $\omega_{i}$ for this purpose.

On the other hand, Figures 9 and 10 depict the time evolution of the stiffness/mass ratios of the vector $\widehat{\Theta}_{K}$, which are estimated through the online LSM (55) with $\delta=\delta_{1}$. Note that these parameters converge at $7.5 \mathrm{~s}$ to a small neighborhood around a constant value. Moreover, Figure $10(\mathrm{~d})$ shows the estimated stiffness $\widehat{K}_{x i}, i=1,2, \ldots, 5$, which are obtained using (64) and the parameter projection scheme in [19]. It is worth mentioning that the initial values employed by the online LSM are $P(0)=1000 I_{75 \times 75}, \widehat{\Theta}_{K}(0)=$ $500[1,1,1, \ldots, 1,1]^{T} \in R^{75 \times 1}$, and $\beta=0.9999$.

5.2. Case in Which Exact Knowledge of Two Natural Frequencies of the Building Is Not Available. If exact knowledge of two 


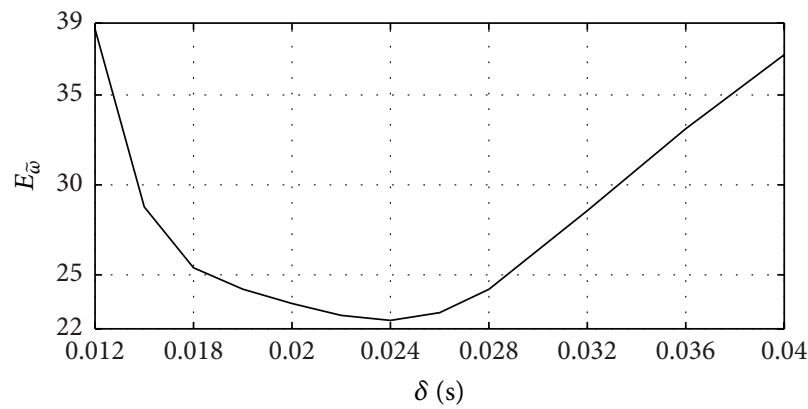

(a) $E_{\widetilde{\omega}}$ versus $\delta$

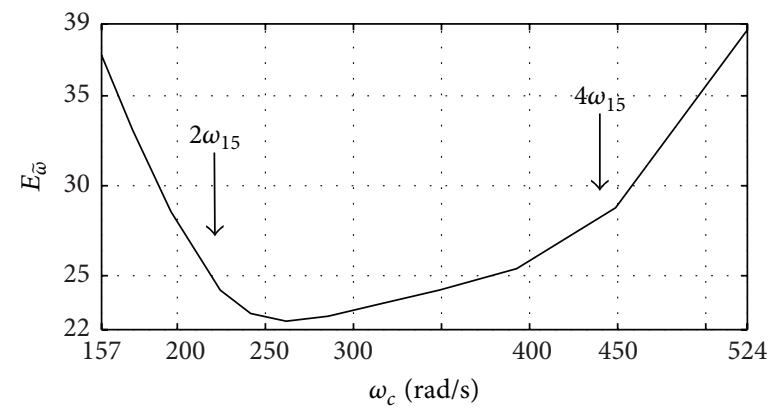

(b) $E_{\widetilde{\omega}}$ versus $\omega_{c}$, where $\omega_{c}=2 \pi / \delta$

Figure 8: Plots of $E_{\widetilde{\omega}}$ versus $\delta$ and $E_{\widetilde{\omega}}$ versus $\omega_{c}$.

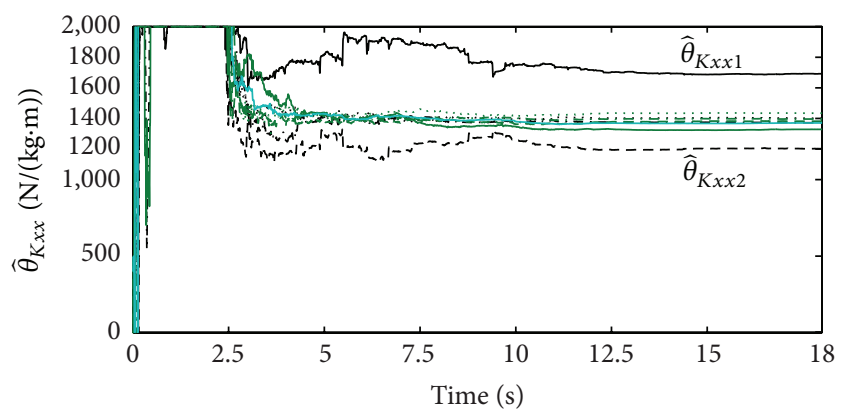

(a) $\widehat{\theta}_{K x x}$

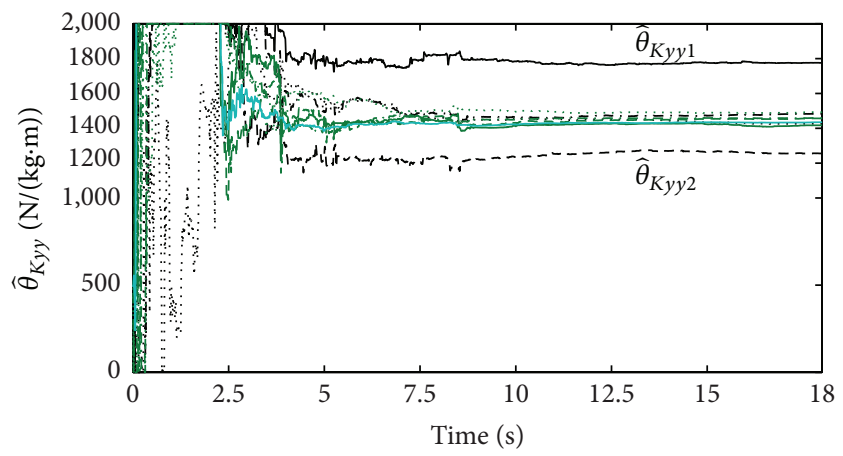

(c) $\hat{\theta}_{K y y}$

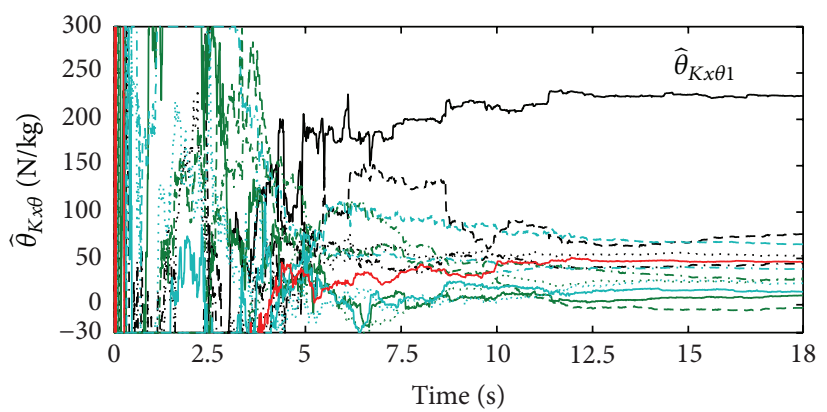

(b) $\widehat{\theta}_{K x \theta}$

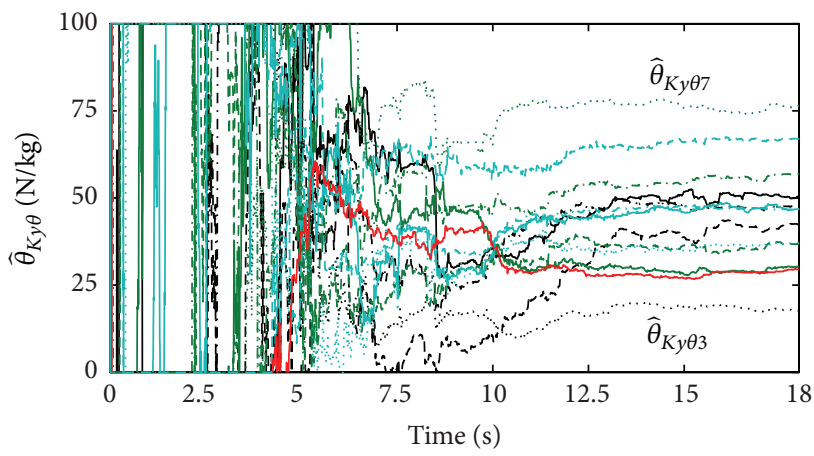

(d) $\hat{\theta}_{K y \theta}$

FIGURE 9: Estimated vectors $\widehat{\theta}_{K x x}, \widehat{\theta}_{K x \theta}, \widehat{\theta}_{K y y}$, and $\widehat{\theta}_{K y \theta}$.

natural frequencies of the building is not available, then the iterative technique shown in Figure 11 can be used to estimate the parameters of the structure, where $L$ is the number of iterations and is generally equal to three or four. Table 6 presents the natural frequencies $\widehat{\omega}_{i}$ and damping ratios $\widehat{\zeta}_{i}$, $i=1,2, \ldots, n$, identified after three iterations, that is, $L=$ 3 , using the offline LSM with $\delta_{1}$ and the initial parameters $\widehat{\omega}_{1 \star}=0.5 \omega_{1}$ and $\widehat{\omega}_{2 \star}=0.5 \omega_{2}$ in (16), which have an error of $50 \%$ with respect to $\omega_{1}$ and $\omega_{2}$. Note that the estimated natural frequencies and damping ratios in Table 6 are similar to those in Tables 2 and 3 for $\delta_{1}$. It is important to mention that the same parameters in Table 6 are obtained if the initial parameters $\widehat{\omega}_{1 \star}$ and $\widehat{\omega}_{2 \star}$ are equal to $\widehat{\omega}_{1 \star}=1.5 \omega_{1}$ and $\widehat{\omega}_{2 \star}=1.5 \omega_{2}$.
5.3. Case in Which the Parameters $\widehat{\omega}_{1 \star}, \widehat{\omega}_{15 \star}, \widehat{\zeta}_{1 \star}$, and $\widehat{\zeta}_{15 \star}$ Are Employed for Computing $a_{0}$ and $a_{1}$ in (16). This section shows the results obtained when the parameters $\widehat{\omega}_{1 \star}, \widehat{\omega}_{15 \star}$, $\widehat{\zeta}_{1 \star}$, and $\widehat{\zeta}_{15 \star}$ are used in (16) for computing the parameters $a_{0}$ and $a_{1}$ of the vector parameterization. Table 7 shows the natural frequencies $\widehat{\omega}_{i}$ and damping ratios $\widehat{\zeta}_{i}, i=1,2, \ldots, 15$, estimated for two cases: one considers that $\widehat{\zeta}_{15 \star}=5 \%$ and the other considers that $\widehat{\zeta}_{15 \star}=10 \%$; in both cases the offline LSM and the parameters $\delta=\delta_{3}, \widehat{\omega}_{1 \star}=\omega_{1}, \widehat{\omega}_{15 \star}=\omega_{15}$, and $\widehat{\zeta}_{1 \star}=1 \%$ are used. Moreover, Figure 12 presents the variation of the index $E_{\widetilde{\omega}}$ with respect to $\widehat{\zeta}_{15 \star}$, which increases from $1 \%$ to $20 \%$, where $\widehat{\zeta}_{1 \star}$ is kept fixed to $1 \%$; note that the best 


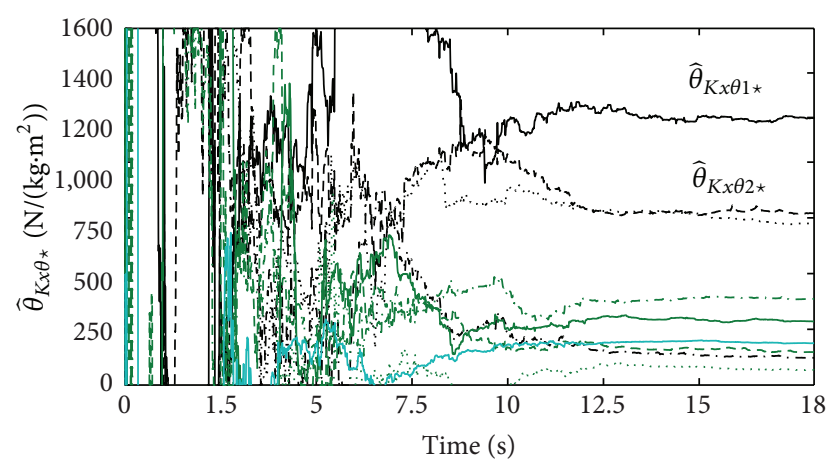

(a) $\hat{\theta}_{K x \theta \star}$

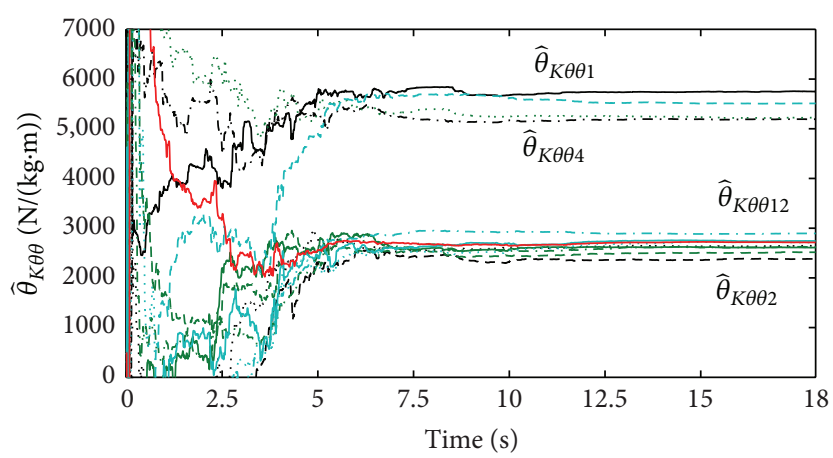

(c) $\widehat{\theta}_{K \theta \theta}$

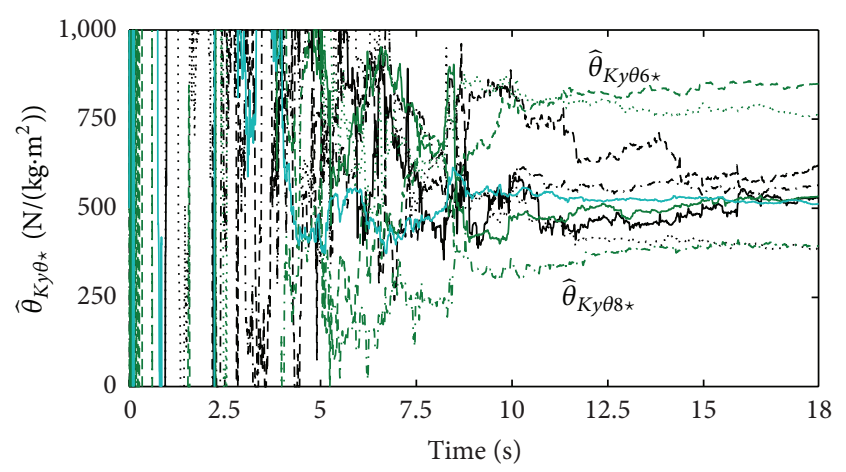

(b) $\widehat{\theta}_{K y \theta *}$

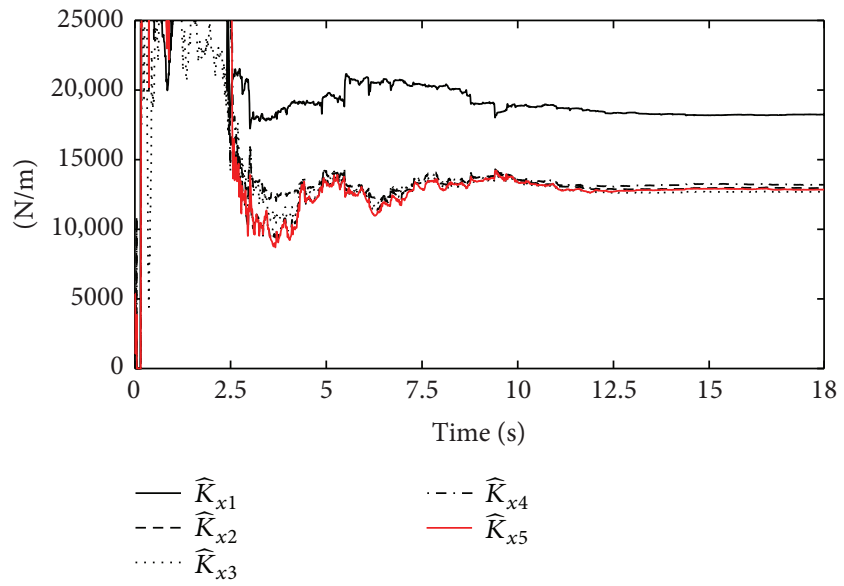

(d) Entries of the matrix $\widehat{K}_{X X}$ in (8)

FIGURE 10: Estimated vectors $\widehat{\theta}_{K x \theta \star}, \widehat{\theta}_{K y \theta \star}$, and $\widehat{\theta}_{K \theta \theta}$, as well as the identified stiffness $\widehat{K}_{x i}, i=1,2, \ldots, 5$.

TABLE 3: Estimated damping ratios.

\begin{tabular}{lll}
\hline $\begin{array}{l}\text { Estimate in } \% \\
\text { with } \delta_{1}\end{array}$ & $\begin{array}{c}\text { Estimate in } \% \\
\text { with } \delta_{2}\end{array}$ & $\begin{array}{c}\text { Estimate in \% } \\
\text { with } \delta_{3}\end{array}$ \\
\hline$\widehat{\zeta}_{1}=1.006$ & $\widehat{\zeta}_{1}=1.005$ & $\widehat{\zeta}_{1}=1.005$ \\
$\widehat{\zeta}_{2}=1.011$ & $\widehat{\zeta}_{2}=1.010$ & $\widehat{\zeta}_{2}=1.009$ \\
$\widehat{\zeta}_{3}=1.084$ & $\widehat{\zeta}_{3}=1.065$ & $\widehat{\zeta}_{3}=1.089$ \\
$\widehat{\zeta}_{4}=1.786$ & $\widehat{\zeta}_{4}=1.781$ & $\widehat{\zeta}_{4}=1.777$ \\
$\widehat{\zeta}_{5}=1.827$ & $\widehat{\zeta}_{5}=1.815$ & $\widehat{\zeta}_{5}=1.804$ \\
$\widehat{\zeta}_{6}=2.437$ & $\widehat{\zeta}_{6}=2.410$ & $\widehat{\zeta}_{6}=2.399$ \\
$\widehat{\zeta}_{7}=2.589$ & $\widehat{\zeta}_{7}=2.580$ & $\widehat{\zeta}_{7}=2.572$ \\
$\widehat{\zeta}_{8}=2.671$ & $\widehat{\zeta}_{8}=2.653$ & $\widehat{\zeta}_{8}=2.640$ \\
$\widehat{\zeta}_{9}=3.251$ & $\widehat{\zeta}_{9}=3.243$ & $\widehat{\zeta}_{9}=3.234$ \\
$\widehat{\zeta}_{10}=3.344$ & $\widehat{\zeta}_{10}=3.325$ & $\widehat{\zeta}_{10}=3.311$ \\
$\widehat{\zeta}_{11}=3.641$ & $\widehat{\zeta}_{11}=3.618$ & $\widehat{\zeta}_{11}=3.594$ \\
$\widehat{\zeta}_{12}=3.728$ & $\widehat{\zeta}_{12}=3.712$ & $\widehat{\zeta}_{12}=3.702$ \\
$\widehat{\zeta}_{13}=3.785$ & $\widehat{\zeta}_{13}=3.761$ & $\widehat{\zeta}_{13}=3.738$ \\
$\widehat{\zeta}_{14}=4.649$ & $\widehat{\zeta}_{14}=4.609$ & $\widehat{\zeta}_{14}=4.565$ \\
$\widehat{\zeta}_{15}=5.211$ & $\widehat{\zeta}_{15}=5.167$ & $\widehat{\zeta}_{15}=5.110$ \\
\hline
\end{tabular}

quality $E_{\widetilde{\omega}}$ of the building model appears when $\widehat{\zeta}_{15 \star}$ takes a value between 1 and $10 \%$. It is worth mentioning that the damping ratios $\widehat{\zeta}_{i}, i=1,2, \ldots, 15$, in Table 7 for $\widehat{\zeta}_{15 \star}=5 \%$ are similar to the ones in Table 3.

5.4. Estimation of the Building Using Reduced Measurements. This section presents the identification of the structure using the vector parameterization, when only some floors are equipped with accelerometers. This estimation procedure that uses reduced measurements has the same objective as the condensation method [18], which provides a reduced degrees of freedom model for structures. Tables 8 and 9 present the estimated natural frequencies and damping ratios of a threestory reduced model, which are obtained when only the first, third, and fifth stories of the building are instrumented and when it is excited through the earthquake in Figure 5. These parameters are computed by means of the offline LSM using $\delta_{4}=0.056 \mathrm{~s}, \delta_{5}=0.06 \mathrm{~s}, \delta_{6}=0.064 \mathrm{~s}$, and the parameters $a_{0}$ and $a_{1}$ in (68). Note that in this reduced measurement case only the first nine natural frequencies of the torsional building are estimated. Table 8 also shows the quality $E_{\widetilde{\omega}}$ of the reduced model, where $n=9$ in (70). In addition, Figure 13 depicts the plots $E_{\widetilde{\omega}}$ versus $\delta$ and $E_{\widetilde{\zeta}}$ versus $\omega_{c}$; from this figure, it is possible to see that the quality of the reduced model is better from $\delta \in[0.051,0.075]$ s. Figure 13(b) also shows the values of $E_{\widetilde{\omega}}$ for $\omega_{c}=1.5 \omega_{9}$ and $\omega_{c}=2 \omega_{9}$, where $\omega_{9}$ is given in Table 1 . It is worth mentioning that the 


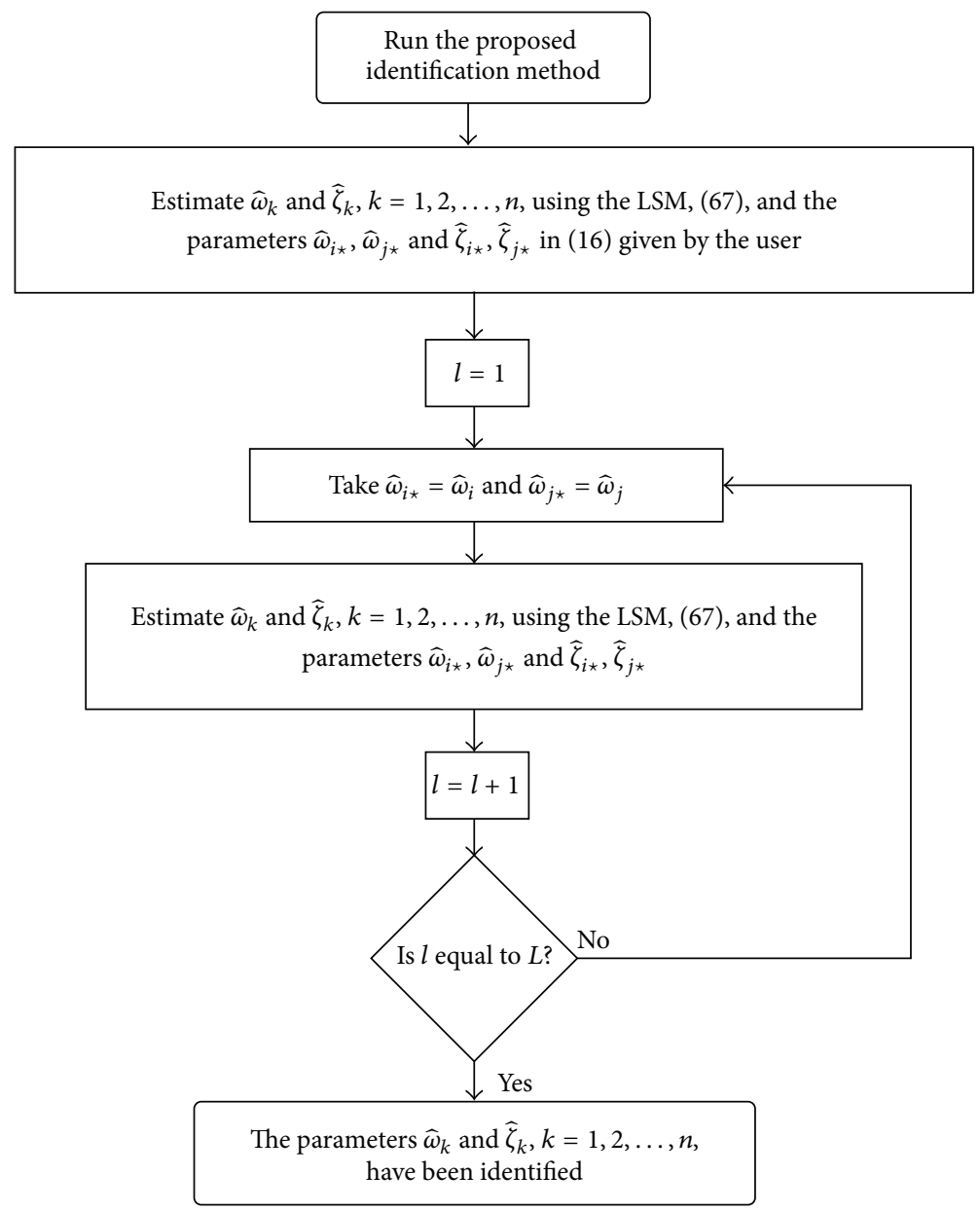

FIGURE 11: Algorithm for estimating the frequencies $\widehat{\omega}_{i}$ and damping ratios $\widehat{\zeta}_{i}, i=1,2, \ldots, n$, of the structure.

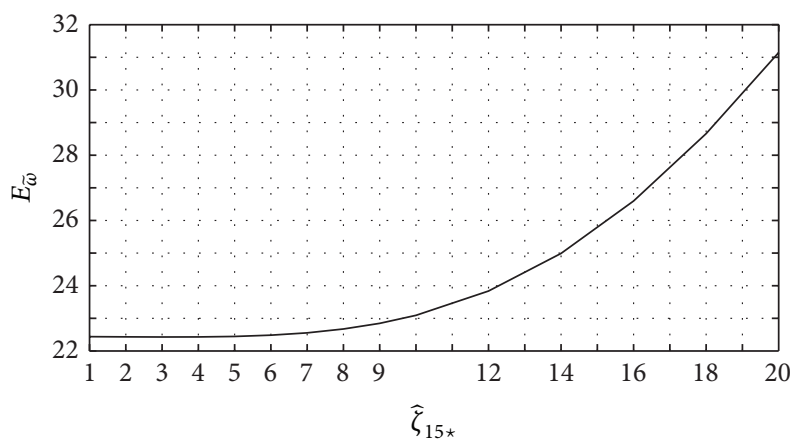

FIGURE 12: $E_{\widetilde{\omega}}$ versus $\widehat{\zeta}_{15 \star}$.

estimated natural frequencies in Table 8 have more errors than the ones in Table 2; however, the identified reduced model is also useful for designing control laws that attenuates the vibrations in the building. Furthermore, a reduced model is sometimes necessary because the number of degrees of freedom of a structure can be very large; and as a consequence, the stiffness, mass, and damping matrices can have large dimensions. In this case, the determination of the natural frequencies and modal shapes is difficult and expensive, and it is desirable to reduce the size of those matrices [18].

On the other hand, a two-story reduced model is identified using only the first and fifth floors' acceleration measurements. Tables 10 and 11 show, respectively, the natural frequencies and damping ratios that are estimated when the offline LSM uses the parameters $\delta_{7}=0.076 \mathrm{~s}, \delta_{8}=0.08 \mathrm{~s}$, and $\delta_{9}=0.084 \mathrm{~s}$; moreover, the parameters $a_{0}$ and $a_{1}$ of the vector parameterization are the ones in (68). The index $E_{\widetilde{\omega}}$ in (70), where $n=6$, is plotted in Figures 14(a) and 14(b) with respect to $\delta \in[0.072,0.1]$ and with respect to $\omega_{c}=2 \pi / \delta$, respectively. From, Figure 14(b) it is possible to see that relative low indexes $E_{\widetilde{\omega}}$ are produced from $\omega_{c}=1.5 \omega_{6}$ to $\omega_{c}=2 \omega_{6}$, where $\omega_{6}$ is presented in Table 1. Finally, the estimated stiffness/mass ratios of the two-story reduced model, obtained with $\delta=$ $\delta_{9} \mathrm{~s}$ and the offline LSM, are presented in Table 12; the corresponding estimates $\widehat{\theta}_{K x x i}$ and $\widehat{\theta}_{K x \theta j}, i=1,2,3, j=$ $1,2,3,4$, produced by the online LSM are shown in Figure 15.

\section{Conclusions}

This paper has presented an identification technique that combines a vector parameterization with the offline or with 


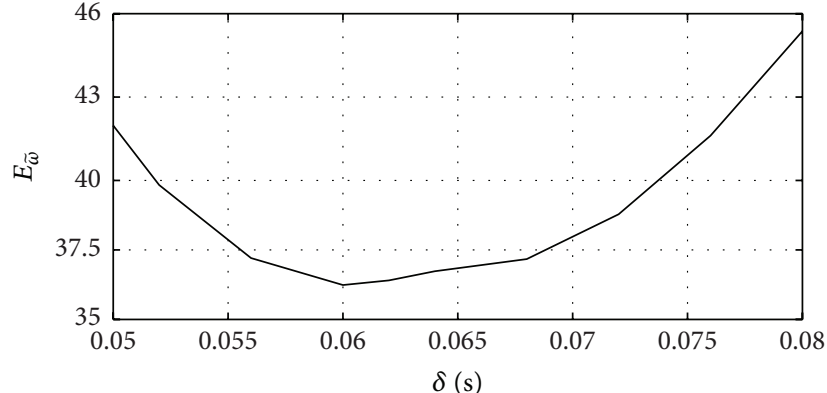

(a) $E_{\widetilde{\omega}}$ versus $\delta$ for the three-story model

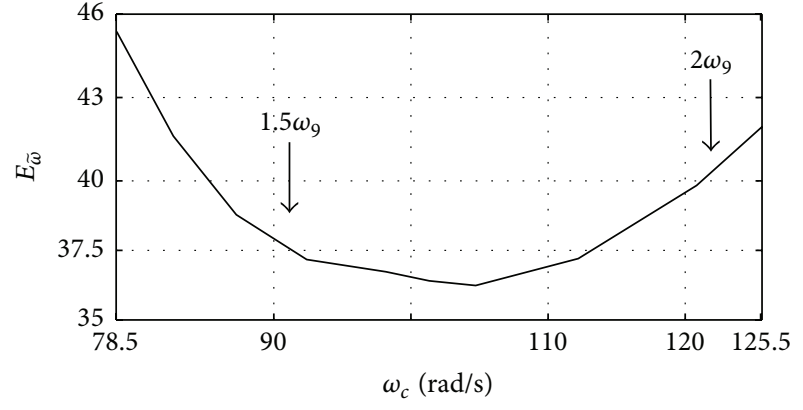

(b) $E_{\widetilde{\omega}}$ versus $\omega_{c}$ for the three-story model

FIGURE 13: Plots of $E_{\widetilde{\omega}}$ versus $\delta$ and $E_{\widetilde{\omega}}$ versus $\omega_{c}$ corresponding to the three-story reduced model.

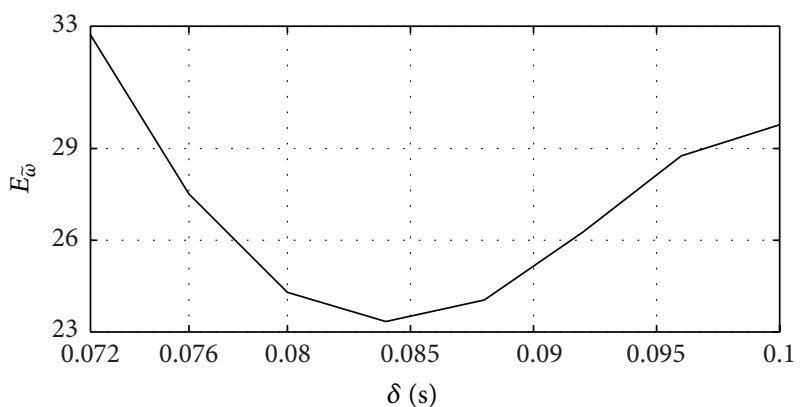

(a) $E_{\widetilde{\omega}}$ versus $\delta$ for the two-story model

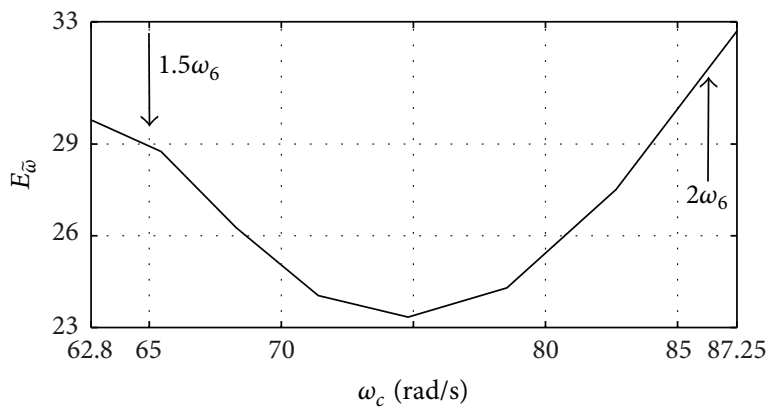

(b) $E_{\widetilde{\omega}}$ versus $\omega_{c}$ for the two-story model

FIGURE 14: Plots of $E_{\widetilde{\omega}}$ versus $\delta$ and $E_{\widetilde{\omega}}$ versus $\omega_{c}$ corresponding to the two-story reduced model.

the online Least Squares Method to recover the parameters of the model of a torsionally coupled building, which is assumed to have classical Rayleigh damping and that is seismically excited. The proposed technique uses acceleration measurements of the floors and ground; it estimates the complete model of the building, as well as its eccentricities, radii of gyration, and the mass, stiffness, and damping matrices, if all the floors are instrumented; and it identifies a reduced model of the building, if only some floors are instrumented. The vector parametrization has the advantage that the number of parameters identified with it is much smaller that the number of parameters identified with a matrix parameterization recently proposed in the literature. The reduction in the number of estimated parameters has a positive impact on the reduction of the computational effort that allows implementing the real-time LSM and has a positive impact on the reduction of the spectral richness of the excitation signal required to uniquely estimate the structural parameters. In addition, the vector parameterization eliminates constant disturbances and attenuates measurement noise by means of Linear Integral Filters (LIF), whose cut-off frequency $\omega_{c}$ is determined by the integration period $\delta$. Experiments using a five-story torsional coupled building mounted on a shaking table were carried in order to verify the performance of the proposed methodology. The results of three tests were presented in the paper; in the first test, the parameters estimated using acceleration measurements of the five floors are obtained; in the remaining two tests, the parameters of three-story and two-story reduced models of the structure are estimated using acceleration measurements of only three and two floors, respectively. An index $E_{\widetilde{\omega}}$ that measures the quality of the models identified during the experiments was also proposed. Experimental results in the full measurement case show that the best quality $E_{\widetilde{\omega}}$ of the identified model is achieved when the frequency $\omega_{c}$ of the LIF is selected such that it takes a value within the interval $\omega_{c} \in\left[2 \omega_{\max }, 4 \omega_{\max }\right]$, where $\omega_{\max }$ is the highest natural frequency of the structure. The experiments also show that when only $n_{r}$ floors are instrumented, the model with the best quality is obtained from $\omega_{c} \in\left[1.5 \omega_{3 * n_{r}}, 2 \omega_{3 * n_{r}}\right]$. Tests also demonstrate that the estimated natural frequencies of the reduced models have more errors than the natural frequencies estimated in the full measurement case; however, a reduced model is also useful for designing control laws that can attenuate vibrations affecting the structure.

\section{Appendix}

\section{A. Parameter Vector $\Theta_{K}$}

The vector $\Theta_{K}$ represents the stiffness/mass ratios of the structure and is defined as

$$
\begin{aligned}
\Theta_{K} & =\left[\theta_{K x x}, \theta_{K x \theta}, \theta_{K y y}, \theta_{K y \theta}, \theta_{K x \theta \star}, \theta_{K y \theta \star}, \theta_{K \theta \theta}\right]^{T} \\
& \in R^{(17 n-10) \times 1},
\end{aligned}
$$




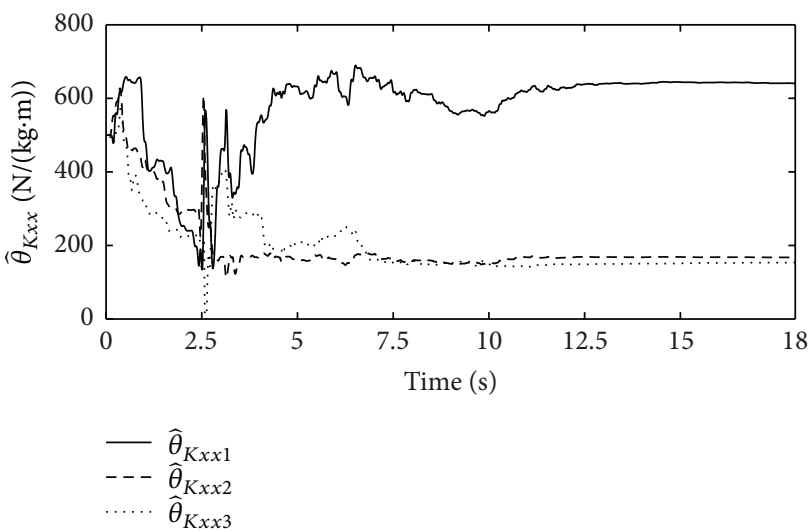

(a) Estimates $\widehat{\theta}_{K x x i}, i=1,2,3$

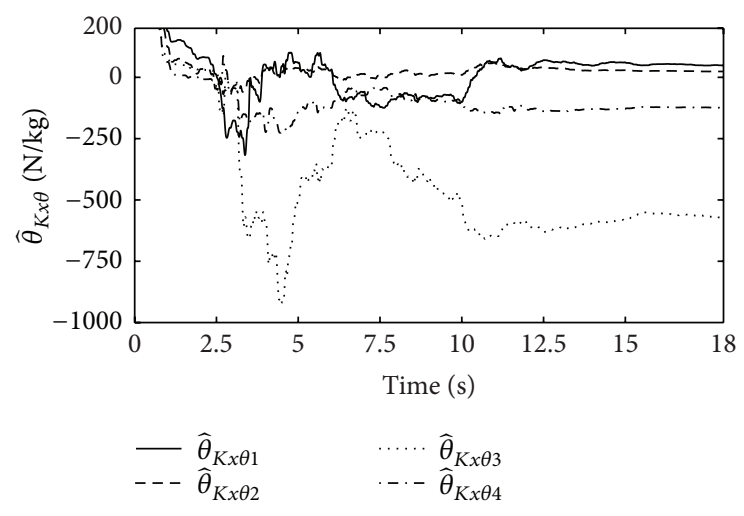

(b) Estimates $\widehat{\theta}_{K x \theta j}, j=1,2,3,4$

FIGURE 15: Estimates $\widehat{\theta}_{K x x i}$ and $\widehat{\theta}_{K x \theta j}, i=1,2,3, j=1,2,3,4$, of the two-story reduced model.

TABLE 4: Nominal and identified eccentricities and radii of gyration.

\begin{tabular}{lc}
\hline $\begin{array}{l}\text { Nominal eccentricities and } \\
\text { radii of gyration }(\mathrm{cm})\end{array}$ & $\begin{array}{c}\text { Identified eccentricities and } \\
\text { radii of gyration }(\mathrm{cm})\end{array}$ \\
\hline$e_{x 1,1}=2.150$ & $\widehat{e}_{x 1,1}=1.970$ \\
$e_{x 1,2}=2.150$ & $\widehat{e}_{x 1,2}=1.240$ \\
$e_{x 2,2}=2.150$ & $\widehat{e}_{x 2,2}=3.400$ \\
$e_{x 2,3}=2.150$ & $\widehat{e}_{x 2,3}=2.620$ \\
$e_{x 3,3}=2.150$ & $\widehat{e}_{x 3,3}=2.050$ \\
$e_{x 3,4}=2.150$ & $\widehat{e}_{x 3,4}=3.140$ \\
$e_{x 4,4}=2.150$ & $\widehat{e}_{x 4,4}=3.920$ \\
$e_{x 4,5}=2.150$ & $\widehat{e}_{x 4,5}=3.270$ \\
$e_{x 5,5}=2.150$ & $\widehat{e}_{x 5,5}=2.550$ \\
$e_{y 1,1}=4.430$ & $\widehat{e}_{y 1,1}=10.660$ \\
$e_{y 1,2}=4.430$ & $\widehat{e}_{y 1,2}=3.790$ \\
$e_{y 2,2}=1.730$ & $\widehat{e}_{y 2,2}=6.360$ \\
$e_{y 2,3}=1.730$ & $\widehat{e}_{y 2,3}=-0.260$ \\
$e_{y 3,3}=1.730$ & $\widehat{e}_{y 3,3}=0.720$ \\
$e_{y 3,4}=1.730$ & $\widehat{e}_{y 3,4}=0.970$ \\
$e_{y 4,4}=1.730$ & $\widehat{e}_{y 4,4}=1.970$ \\
$e_{y 4,5}=1.730$ & $\widehat{e}_{y 4,5}=2.820$ \\
$e_{y 5,5}=1.730$ & $\widehat{e}_{y 5,5}=1.670$ \\
$r_{1}=25.050$ & $\widehat{r}_{1}=25.690$ \\
$r_{2}=24.910$ & $\widehat{r}_{2}=26.310$ \\
$r_{3}=24.910$ & $\widehat{r}_{3}=23.380$ \\
$r_{4}=24.910$ & $\widehat{r}_{4}=27.760$ \\
$r_{5}=24.910$ & $\widehat{r}_{5}=26.750$ \\
&
\end{tabular}

$$
\begin{aligned}
& \theta_{K x x}=\left[\theta_{K x x 1}, \theta_{K x x 2}, \theta_{K x x 3}, \ldots, \theta_{K x x(2 n-1)}\right]^{T} \\
& \in R^{(2 n-1) \times 1} \\
& =\left[\frac{K_{x 1}}{m_{1}}, \frac{K_{x 2}}{m_{1}}, \frac{K_{x 2}}{m_{2}}, \frac{K_{x 3}}{m_{2}}, \frac{K_{x 3}}{m_{3}}, \ldots, \frac{K_{x n}}{m_{n}}\right]^{T},
\end{aligned}
$$

$$
\begin{aligned}
& \theta_{K x \theta}=\left[\theta_{K x \theta 1}, \theta_{K x \theta 2}, \theta_{K x \theta 3}, \ldots, \theta_{K x \theta(3 n-2)}\right]^{T} \\
& \in R^{(3 n-2) \times 1} \\
& =\left[\frac{e_{y 1,1} K_{x 1}+e_{y 1,2} K_{x 2}}{m_{1}}, \frac{e_{y 2,2} K_{x 2}}{m_{1}}, \frac{e_{y 1,2} K_{x 2}}{m_{2}},\right. \\
& \left.\frac{e_{y 2,2} K_{x 2}+e_{y 2,3} K_{x 3}}{m_{2}}, \frac{e_{y 3,3} K_{x 3}}{m_{2}}, \ldots, \frac{e_{y n, n} K_{x n}}{m_{n}}\right]^{T}, \\
& \theta_{K y y}=\left[\theta_{K y y 1}, \theta_{K y y 2}, \theta_{K y y 3}, \ldots, \theta_{K y y(2 n-1)}\right]^{T} \\
& \in R^{(2 n-1) \times 1} \\
& =\left[\frac{K_{y 1}}{m_{1}}, \frac{K_{y 2}}{m_{1}}, \frac{K_{y 2}}{m_{2}}, \frac{K_{y 3}}{m_{2}}, \frac{K_{y 3}}{m_{3}}, \ldots, \frac{K_{y n}}{m_{n}}\right]^{T}, \\
& \theta_{K y \theta}=\left[\theta_{K y \theta 1}, \theta_{K y \theta 2}, \theta_{K y \theta 3}, \ldots, \theta_{K y \theta(3 n-2)}\right]^{T} \\
& \in R^{(3 n-2) \times 1} \\
& =\left[\frac{e_{x 1,1} K_{y 1}+e_{x 1,2} K_{y 2}}{m_{1}}, \frac{e_{x 2,2} K_{y 2}}{m_{1}}, \frac{e_{x 1,2} K_{y 2}}{m_{2}},\right. \\
& \left.\frac{e_{x 2,2} K_{y 2}+e_{x 2,3} K_{y 3}}{m_{2}}, \frac{e_{x 3,3} K_{y 3}}{m_{2}}, \ldots, \frac{e_{x n, n} K_{y n}}{m_{n}}\right]^{T} \text {, } \\
& \theta_{K x \theta \star}=\left[\theta_{K x \theta 1 \star}, \theta_{K x \theta 2 \star}, \theta_{K x \theta 3 \star}, \ldots, \theta_{K x \theta(2 n-1) \star}\right]^{T} \\
& \in R^{(2 n-1) \times 1} \\
& =\left[\frac{e_{y 1,1} K_{x 1}}{m_{1} r_{1}^{2}}, \frac{e_{y 1,2} K_{x 2}}{m_{1} r_{1}^{2}}, \frac{e_{y 2,2} K_{x 2}}{m_{2} r_{2}^{2}}, \frac{e_{y 2,3} K_{x 3}}{m_{2} r_{2}^{2}}, \frac{e_{y 3,3} K_{x 3}}{m_{3} r_{3}^{2}},\right. \\
& \left.\ldots, \frac{e_{y n, n} K_{x n}}{m_{n} r_{n}^{2}}\right]^{T}
\end{aligned}
$$

(A.2) 
TABLE 5: Nominal and identified masses, stiffness, and natural frequencies.

\begin{tabular}{|c|c|c|c|c|c|}
\hline $\begin{array}{l}\text { Nominal mass } \\
(\mathrm{kg})\end{array}$ & $\begin{array}{l}\text { Estimated mass } \\
(\mathrm{kg})\end{array}$ & $\begin{array}{c}\text { Nominal stiffness } \\
(\mathrm{kN} / \mathrm{m})\end{array}$ & $\begin{array}{c}\text { Estimated stiffness } \\
(\mathrm{kN} / \mathrm{m})\end{array}$ & $\begin{array}{c}\underset{\operatorname{Nominal} \omega_{i}^{\text {nom }}}{(\mathrm{rad} / \mathrm{s})} \\
\end{array}$ & $\begin{array}{c}\text { Estimated } \widehat{\omega}_{i} \\
(\mathrm{rad} / \mathrm{s})\end{array}$ \\
\hline$m_{1}=10.780$ & $\widehat{m}_{1}=10.780$ & $K_{x 1}=18.415$ & $\widehat{K}_{x 1}=18.240$ & $\omega_{1}^{\text {nom }}=11.012$ & $\widehat{\omega}_{1}=10.948$ \\
\hline$m_{2}=9.200$ & $\widehat{m}_{2}=9.250$ & $K_{x 2}=12.336$ & $\widehat{K}_{x 2}=12.959$ & $\omega_{2}^{\text {nom }}=11.065$ & $\widehat{\omega}_{2}=11.372$ \\
\hline$m_{3}=9.200$ & $\widehat{m}_{3}=9.550$ & $K_{x 3}=12.336$ & $\widehat{K}_{x 3}=12.688$ & $\omega_{3}^{\text {nom }}=16.780$ & $\widehat{\omega}_{3}=14.696$ \\
\hline$m_{4}=9.200$ & $\widehat{m}_{4}=9.200$ & $K_{x 4}=12.336$ & $\widehat{K}_{x 4}=13.193$ & $\omega_{4}^{\text {nom }}=31.607$ & $\widehat{\omega}_{4}=31.937$ \\
\hline \multirow[t]{15}{*}{$m_{5}=9.200$} & $\widehat{m}_{5}=9.370$ & $K_{x 5}=12.336$ & $\widehat{K}_{x 5}=12.845$ & $\omega_{5}^{\text {nom }}=31.794$ & $\widehat{\omega}_{5}=32.834$ \\
\hline & & $K_{y 1}=18.415$ & $\widehat{K}_{y 1}=19.144$ & $\omega_{6}^{\text {nom }}=47.635$ & $\widehat{\omega}_{6}=45.563$ \\
\hline & & $K_{y 2}=12.336$ & $\widehat{K}_{y 2}=13.539$ & $\omega_{7}^{\text {nom }}=48.678$ & $\widehat{\omega}_{7}=48.677$ \\
\hline & & $K_{y 3}=12.336$ & $\widehat{K}_{y 3}=13.719$ & $\omega_{8}^{\text {nom }}=49.133$ & $\widehat{\omega}_{8}=50.344$ \\
\hline & & $K_{y 4}=12.336$ & $\widehat{K}_{y 4}=13.908$ & $\omega_{9}^{\text {nom }}=61.157$ & $\widehat{\omega}_{9}=62.049$ \\
\hline & & $K_{y 5}=12.336$ & $\widehat{K}_{y 5}=13.420$ & $\omega_{10}^{\text {nom }}=61.612$ & $\widehat{\omega}_{10}=63.904$ \\
\hline & & $K_{91}=4.380$ & $\widehat{K}_{91}=4.299$ & $\omega_{11}^{\text {nom }}=69.700$ & $\widehat{\omega}_{11}=69.844$ \\
\hline & & $K_{92}=1.742$ & $\widehat{K}_{92}=1.751$ & $\omega_{12}^{\text {nom }}=70.082$ & $\widehat{\omega}_{12}=71.580$ \\
\hline & & $K_{93}=3.473$ & $\widehat{K}_{93}=3.444$ & $\omega_{13}^{\text {nom }}=74.250$ & $\widehat{\omega}_{13}=72.729$ \\
\hline & & $K_{94}=1.737$ & $\widehat{K}_{94}=1.680$ & $\omega_{14}^{\text {nom }}=93.386$ & $\widehat{\omega}_{14}=89.871$ \\
\hline & & $K_{95}=3.473$ & $\widehat{K}_{95}=2.833$ & $\omega_{15}^{\text {nom }}=106.192$ & $\widehat{\omega}_{15}=100.990$ \\
\hline & & $K_{96}=1.737$ & $\widehat{K}_{96}=1.484$ & & \\
\hline & & $K_{97}=3.473$ & $\widehat{K}_{97}=4.048$ & & \\
\hline & & $K_{98}=1.737$ & $\widehat{K}_{98}=1.892$ & & \\
\hline & & $K_{\vartheta 9}=1.737$ & $\widehat{K}_{99}=1.815$ & & \\
\hline
\end{tabular}

$$
\begin{aligned}
& \theta_{K y \theta \star}=\left[\theta_{K y \theta 1 \star}, \theta_{K y \theta 2 \star}, \theta_{K y \theta 3 \star}, \ldots, \theta_{K y \theta(2 n-1) \star}\right]^{T} \\
& \in R^{(2 n-1) \times 1} \\
& =\left[\frac{e_{x 1,1} K_{y 1}}{m_{1} r_{1}^{2}}, \frac{e_{x 1,2} K_{y 2}}{m_{1} r_{1}^{2}}, \frac{e_{x 2,2} K_{y 2}}{m_{2} r_{2}^{2}}, \frac{e_{x 2,3} K_{y 3}}{m_{2} r_{2}^{2}}, \frac{e_{x 3,3} K_{y 3}}{m_{3} r_{3}^{2}},\right. \\
& \left.\quad \ldots, \frac{e_{x n, n} K_{y n}}{m_{n} r_{n}^{2}}\right]^{T}, \\
& \theta_{K \theta \theta}=\left[\theta_{K \theta \theta 1}, \theta_{K \theta \theta 2}, \theta_{K \theta \theta 3}, \ldots, \theta_{K \theta \theta(3 n-2)}\right]^{T} \\
& \in R^{(3 n-2) \times 1} \\
& =\left[\frac{K_{91}}{m_{1} r_{1}^{2}}, \frac{K_{92}}{m_{1} r_{1}^{2}}, \frac{K_{92}}{m_{2} r_{2}^{2}}, \frac{K_{93}}{m_{2} r_{2}^{2}}, \frac{K_{94}}{m_{2} r_{2}^{2}}, \ldots, \frac{K_{\vartheta(2 n-1)}}{m_{n} r_{n}^{2}}\right]^{T} .
\end{aligned}
$$

\section{B. Proof of Proposition 4}

Proof. Using (43), the variable $I_{5}[r]$ can be expressed in the frequency domain as

$$
\mathscr{L}\left(I_{5}[r]\right)=\frac{1}{\delta^{4}}\left(\frac{1-e^{-\delta s}}{s}\right)^{5} R(s)
$$

TABLE 6: Natural frequencies and damping ratios estimated with the algorithm in Figure 11, where $L=3$ and the initial frequencies $\widehat{\omega}_{1 \star}$ and $\widehat{\omega}_{2 \star}$ in (16) have an error of $50 \%$ with respect to $\omega_{1}$ and $\omega_{2}$.

\begin{tabular}{lcc}
\hline$\widehat{\omega}(\mathrm{rad} / \mathrm{s})$ & $\widetilde{\omega}(\%)$ & $\widehat{\zeta}(\%)$ \\
\hline$\widehat{\omega}_{1}=10.943$ & $\widetilde{\omega}_{1}=14.133$ & $\widehat{\zeta}_{1}=1.000$ \\
$\widehat{\omega}_{2}=11.382$ & $\widetilde{\omega}_{2}=14.143$ & $\widehat{\zeta}_{2}=1.000$ \\
$\widehat{\omega}_{3}=14.570$ & $\widetilde{\omega}_{3}=0.647$ & $\widehat{\zeta}_{3}=1.036$ \\
$\widehat{\omega}_{4}=31.950$ & $\widetilde{\omega}_{4}=7.165$ & $\widehat{\zeta}_{4}=1.606$ \\
$\widehat{\omega}_{5}=32.841$ & $\widetilde{\omega}_{5}=6.714$ & $\widehat{\zeta}_{5}=1.641$ \\
$\widehat{\omega}_{6}=45.558$ & $\widetilde{\omega}_{6}=5.131$ & $\widehat{\zeta}_{6}=2.163$ \\
$\widehat{\omega}_{7}=48.693$ & $\widetilde{\omega}_{7}=5.366$ & $\widehat{\zeta}_{7}=2.296$ \\
$\widehat{\omega}_{8}=50.367$ & $\widetilde{\omega}_{8}=4.024$ & $\widehat{\zeta}_{8}=2.367$ \\
$\widehat{\omega}_{9}=62.085$ & $\widetilde{\omega}_{9}=1.814$ & $\widehat{\zeta}_{9}=2.871$ \\
$\widehat{\omega}_{10}=63.934$ & $\widetilde{\omega}_{10}=1.449$ & $\widehat{\zeta}_{10}=2.951$ \\
$\widehat{\omega}_{11}=69.890$ & $\widetilde{\omega}_{11}=1.956$ & $\widehat{\zeta}_{11}=3.210$ \\
$\widehat{\omega}_{12}=71.620$ & $\widetilde{\omega}_{12}=0.535$ & $\widetilde{\zeta}_{12}=3.286$ \\
$\widehat{\omega}_{13}=72.771$ & $\widetilde{\omega}_{13}=0.624$ & $\widetilde{\zeta}_{13}=3.336$ \\
$\widehat{\omega}_{14}=89.967$ & $\widetilde{\omega}_{14}=0.357$ & $\widehat{\zeta}_{14}=4.092$ \\
$\widehat{\omega}_{15}=101.091$ & $\widetilde{\omega}_{15}=8.167$ & $\widehat{\zeta}_{15}=4.583$ \\
& $E_{\widetilde{\omega}}=25.390$ & \\
\hline
\end{tabular}


TABLE 7: Estimated natural frequencies obtained using estimates $\widehat{\omega}_{1 \star}=\omega_{1}, \widehat{\omega}_{15 \star}=\omega_{15}, \widehat{\zeta}_{1 \star}$, and $\widehat{\zeta}_{15 \star}$.

\begin{tabular}{|c|c|c|c|c|c|}
\hline \multicolumn{3}{|c|}{$\widehat{\zeta}_{1 \star}=1 \%$ and $\widehat{\zeta}_{15 \star}=5 \%$} & \multicolumn{3}{|c|}{$\widehat{\zeta}_{1 \star}=1 \%$ and $\widehat{\zeta}_{15 \star}=10 \%$} \\
\hline$\widehat{\omega}(\mathrm{rad} / \mathrm{s})$ & $\widetilde{\omega}(\%)$ & $\widehat{\zeta}(\%)$ & $\widehat{\omega}(\mathrm{rad} / \mathrm{s})$ & $\widetilde{\omega}(\%)$ & $\widehat{\zeta}(\%)$ \\
\hline$\widehat{\omega}_{1}=10.750$ & $\widetilde{\omega}_{1}=12.115$ & $\widehat{\zeta}_{1}=0.991$ & $\widehat{\omega}_{1}=10.780$ & $\widetilde{\omega}_{1}=12.430$ & $\widehat{\zeta}_{1}=1.094$ \\
\hline$\widehat{\omega}_{2}=11.156$ & $\widetilde{\omega}_{2}=11.885$ & $\widehat{\zeta}_{2}=0.991$ & $\widehat{\omega}_{2}=11.119$ & $\widetilde{\omega}_{2}=11.506$ & $\widehat{\zeta}_{2}=1.121$ \\
\hline$\widehat{\omega}_{3}=14.515$ & $\widetilde{\omega}_{3}=0.264$ & $\widehat{\zeta}_{3}=1.028$ & $\widehat{\omega}_{3}=15.127$ & $\widetilde{\omega}_{3}=4.491$ & $\widehat{\zeta}_{3}=1.455$ \\
\hline$\widehat{\omega}_{4}=31.774$ & $\widetilde{\omega}_{4}=6.577$ & $\widehat{\zeta}_{4}=1.601$ & $\widehat{\omega}_{4}=31.672$ & $\widetilde{\omega}_{4}=6.232$ & $\widehat{\zeta}_{4}=2.913$ \\
\hline$\widehat{\omega}_{5}=32.352$ & $\widetilde{\omega}_{5}=5.125$ & $\widehat{\zeta}_{5}=1.623$ & $\widehat{\omega}_{5}=32.261$ & $\widetilde{\omega}_{5}=4.829$ & $\widehat{\zeta}_{5}=2.966$ \\
\hline$\widehat{\omega}_{6}=44.641$ & $\widetilde{\omega}_{6}=3.014$ & $\widehat{\zeta}_{6}=2.130$ & $\widehat{\omega}_{6}=44.574$ & $\widetilde{\omega}_{6}=2.858$ & $\widehat{\zeta}_{6}=4.072$ \\
\hline$\widehat{\omega}_{7}=48.381$ & $\widetilde{\omega}_{7}=4.693$ & $\widehat{\zeta}_{7}=2.288$ & $\widehat{\omega}_{7}=48.233$ & $\widetilde{\omega}_{7}=4.371$ & $\widehat{\zeta}_{7}=4.402$ \\
\hline$\widehat{\omega}_{8}=49.699$ & $\widetilde{\omega}_{8}=2.646$ & $\widehat{\zeta}_{8}=2.345$ & $\widehat{\omega}_{8}=49.481$ & $\widetilde{\omega}_{8}=2.195$ & $\widehat{\zeta}_{8}=4.515$ \\
\hline$\widehat{\omega}_{9}=61.761$ & $\widetilde{\omega}_{9}=1.284$ & $\widehat{\zeta}_{9}=2.866$ & $\widehat{\omega}_{9}=61.441$ & $\widetilde{\omega}_{9}=0.759$ & $\widehat{\zeta}_{9}=5.595$ \\
\hline$\widehat{\omega}_{10}=63.223$ & $\widetilde{\omega}_{10}=0.322$ & $\widehat{\zeta}_{10}=2.929$ & $\widehat{\omega}_{10}=62.942$ & $\widetilde{\omega}_{10}=0.124$ & $\widehat{\zeta}_{10}=5.731$ \\
\hline$\widehat{\omega}_{11}=68.954$ & $\widetilde{\omega}_{11}=0.591$ & $\widehat{\zeta}_{11}=3.180$ & $\widehat{\omega}_{11}=68.538$ & $\widetilde{\omega}_{11}=0.016$ & $\widehat{\zeta}_{11}=6.237$ \\
\hline$\widehat{\omega}_{12}=71.051$ & $\widetilde{\omega}_{12}=1.325$ & $\widehat{\zeta}_{12}=3.272$ & $\widehat{\omega}_{12}=70.637$ & $\widetilde{\omega}_{12}=1.900$ & $\widehat{\zeta}_{12}=6.427$ \\
\hline$\widehat{\omega}_{13}=71.826$ & $\widetilde{\omega}_{13}=0.682$ & $\widehat{\zeta}_{13}=3.306$ & $\widehat{\omega}_{13}=71.460$ & $\widetilde{\omega}_{13}=1.188$ & $\widehat{\zeta}_{13}=6.502$ \\
\hline$\widehat{\omega}_{14}=88.363$ & $\widetilde{\omega}_{14}=2.133$ & $\widehat{\zeta}_{14}=4.035$ & $\widehat{\omega}_{14}=87.506$ & $\widetilde{\omega}_{14}=3.082$ & $\widehat{\zeta}_{14}=7.954$ \\
\hline \multirow[t]{2}{*}{$\widehat{\omega}_{15}=99.118$} & $\widetilde{\omega}_{15}=9.959$ & $\widehat{\zeta}_{15}=4.512$ & $\widehat{\omega}_{15}=98.185$ & $\widetilde{\omega}_{15}=10.807$ & $\widehat{\zeta}_{15}=8.922$ \\
\hline & $E_{\widetilde{\omega}}=22.445$ & & & $E_{\widetilde{\omega}}=23.091$ & \\
\hline
\end{tabular}

TABLE 8: Estimated natural frequencies of the three-story reduced model.

\begin{tabular}{lcccccc}
\hline $\begin{array}{l}\text { Natural frequency } \\
\text { (rad/s) }\end{array}$ & Estimate with $\delta_{4}$ & Error (\%) & Estimate with $\delta_{5}$ & Error (\%) & Estimate with $\delta_{6}$ & Error $(\%)$ \\
\hline$\omega_{1}=9.588$ & 11.481 & $\widetilde{\omega}_{1}=19.745$ & 11.278 & $\widetilde{\omega}_{1}=17.622$ & 11.080 & $\widetilde{\omega}_{1}=15.559$ \\
$\omega_{2}=9.971$ & 12.322 & $\widetilde{\omega}_{2}=23.576$ & 11.941 & $\widetilde{\omega}_{2}=19.757$ & 11.603 & $\widetilde{\omega}_{2}=16.365$ \\
$\omega_{3}=14.476$ & 15.034 & $\widetilde{\omega}_{3}=3.850$ & 15.074 & $\widetilde{\omega}_{3}=4.126$ & 15.003 & $\widetilde{\omega}_{3}=3.637$ \\
$\omega_{4}=29.814$ & 32.595 & $\widetilde{\omega}_{4}=9.328$ & 31.911 & $\widetilde{\omega}_{4}=7.034$ & 31.289 & 32.285 \\
$\omega_{5}=30.775$ & 33.711 & $\widetilde{\omega}_{5}=9.540$ & 32.981 & $\widetilde{\omega}_{5}=7.167$ & $\widetilde{\omega}_{4}=4.950$ \\
$\omega_{6}=43.335$ & 42.174 & $\widetilde{\omega}_{6}=2.678$ & 41.439 & $\widetilde{\omega}_{6}=4.375$ & 40.964 & $\widetilde{\omega}_{6}=5.470$ \\
$\omega_{7}=46.213$ & 43.853 & $\widetilde{\omega}_{7}=5.106$ & 42.939 & $\widetilde{\omega}_{7}=7.084$ & 42.129 & $\widetilde{\omega}_{7}=8.837$ \\
$\omega_{8}=48.418$ & 45.882 & $\widetilde{\omega}_{8}=5.237$ & 45.563 & $\widetilde{\omega}_{8}=5.897$ & 45.570 & $\widetilde{\omega}_{8}=5.883$ \\
$\omega_{9}=60.978$ & 52.682 & $\widetilde{\omega}_{9}=13.605$ & 48.931 & $\widetilde{\omega}_{9}=19.757$ & 45.613 & $\widetilde{\omega}_{9}=25.198$ \\
& & $E_{\widetilde{\omega}}=37.206$ & & $E_{\widetilde{\omega}}=36.238$ & & $E_{\widetilde{\omega}}=36.732$ \\
\hline
\end{tabular}

TABLE 9: Estimated damping ratios of the three-story reduced model.

\begin{tabular}{lcc}
\hline $\begin{array}{l}\text { Estimate in \% } \\
\text { with } \delta_{1}\end{array}$ & $\begin{array}{c}\text { Estimate in \% } \\
\text { with } \delta_{2}\end{array}$ & $\begin{array}{c}\text { Estimate in \% } \\
\text { with } \delta_{3}\end{array}$ \\
\hline$\widehat{\zeta}_{1}=1.006$ & $\widehat{\zeta}_{1}=1.010$ & $\widehat{\zeta}_{1}=0.308$ \\
$\widehat{\zeta}_{2}=1.011$ & $\widehat{\zeta}_{2}=1.020$ & $\widehat{\zeta}_{2}=1.008$ \\
$\widehat{\zeta}_{3}=1.084$ & $\widehat{\zeta}_{3}=1.095$ & $\widehat{\zeta}_{3}=1.014$ \\
$\widehat{\zeta}_{4}=1.786$ & $\widehat{\zeta}_{4}=1.785$ & $\widehat{\zeta}_{4}=1.093$ \\
$\widehat{\zeta}_{5}=1.827$ & $\widehat{\zeta}_{5}=1.834$ & $\widehat{\zeta}_{5}=1.756$ \\
$\widehat{\zeta}_{6}=2.437$ & $\widehat{\zeta}_{6}=2.236$ & $\widehat{\zeta}_{6}=1.802$ \\
$\widehat{\zeta}_{7}=2.589$ & $\widehat{\zeta}_{7}=2.309$ & $\widehat{\zeta}_{7}=2.214$ \\
$\widehat{\zeta}_{8}=2.671$ & $\widehat{\zeta}_{8}=2.437$ & $\widehat{\zeta}_{8}=2.270$ \\
$\widehat{\zeta}_{9}=3.251$ & $\widehat{\zeta}_{9}=2.601$ & $\widehat{\zeta}_{9}=4.566$ \\
\hline
\end{tabular}

Substituting $R(s)$ from (38) into (B.1) produces

$$
\begin{gathered}
\mathscr{L}\left(I_{5}[r]\right)=\frac{1}{\delta^{4}}\left[-\gamma \Theta_{K} \frac{\left(1-e^{-\delta s}\right)^{5}}{s^{5}}\right. \\
+\left(a_{0} D-a_{1} \gamma \Theta_{K}\right) \frac{\left(1-e^{-\delta s}\right)^{5}}{s^{4}} \\
\left.+\left[D+D_{g}\right] \frac{\left(1-e^{-\delta s}\right)^{5}}{s^{3}}\right] .
\end{gathered}
$$


TABLE 10: Estimated natural frequencies of the two-story reduced model.

\begin{tabular}{lcccccc}
\hline Natural frequency $(\mathrm{rad} / \mathrm{s})$ & Estimate with $\delta_{7}$ & Error $(\%)$ & Estimate with $\delta_{8}$ & Error $(\%)$ & Estimate with $\delta_{9}$ & Error $(\%)$ \\
\hline$\omega_{1}=9.588$ & 11.096 & $\widetilde{\omega}_{1}=15.731$ & 10.965 & $\widetilde{\omega}_{1}=14.356$ & 10.976 & $\widetilde{\omega}_{1}=14.475$ \\
$\omega_{2}=9.971$ & 11.895 & $\widetilde{\omega}_{2}=19.294$ & 11.452 & $\widetilde{\omega}_{2}=14.849$ & 10.976 & $\widetilde{\omega}_{2}=10.077$ \\
$\omega_{3}=14.476$ & 15.331 & $\widetilde{\omega}_{3}=5.905$ & 15.105 & $\widetilde{\omega}_{3}=4.342$ & 14.932 & $\widetilde{\omega}_{3}=3.147$ \\
$\omega_{4}=29.814$ & 30.590 & $\widetilde{\omega}_{4}=2.603$ & 29.919 & $\widetilde{\omega}_{4}=0.355$ & 29.284 & $\widetilde{\omega}_{4}=1.778$ \\
$\omega_{5}=30.775$ & 30.904 & $\widetilde{\omega}_{5}=0.420$ & 30.479 & $\widetilde{\omega}_{5}=0.963$ & 30.447 & $\widetilde{\omega}_{5}=1.065$ \\
$\omega_{6}=43.335$ & 39.101 & $\widetilde{\omega}_{6}=9.770$ & 38.139 & $\widetilde{\omega}_{6}=11.990$ & 36.915 & $\widetilde{\omega}_{6}=14.815$ \\
& & $E_{\widetilde{\omega}}=27.514$ & & $E_{\widetilde{\omega}}=24.295$ & & \\
\hline
\end{tabular}

TABLE 11: Estimated damping ratios of the two-story reduced model.

\begin{tabular}{lcc}
\hline $\begin{array}{l}\text { Estimate in \% } \\
\text { with } \delta_{7}\end{array}$ & $\begin{array}{c}\text { Estimate in \% } \\
\text { with } \delta_{8}\end{array}$ & $\begin{array}{c}\text { Estimate in \% } \\
\text { with } \delta_{9}\end{array}$ \\
\hline$\widehat{\zeta}_{1}=1.008$ & $\widehat{\zeta}_{1}=1.006$ & $\widehat{\zeta}_{1}=0.305$ \\
$\widehat{\zeta}_{2}=1.019$ & $\widehat{\zeta}_{2}=1.012$ & $\widehat{\zeta}_{2}=1.091$ \\
$\widehat{\zeta}_{3}=1.103$ & $\widehat{\zeta}_{3}=1.096$ & $\widehat{\zeta}_{3}=1.664$ \\
$\widehat{\zeta}_{4}=1.724$ & $\widehat{\zeta}_{4}=1.693$ & $\widehat{\zeta}_{4}=1.708$ \\
$\widehat{\zeta}_{5}=1.738$ & $\widehat{\zeta}_{5}=1.719$ & $\widehat{\zeta}_{5}=1.717$ \\
$\widehat{\zeta}_{6}=2.124$ & $\widehat{\zeta}_{6}=2.078$ & $\widehat{\zeta}_{6}=2.020$ \\
\hline
\end{tabular}

TABLE 12: Stiffness/mass ratios of the two-story reduced model.

\begin{tabular}{lccc}
\hline $\mathrm{N} /(\mathrm{kg} \cdot \mathrm{m})$ & $\mathrm{N} / \mathrm{kg}$ & $\mathrm{N} /\left(\mathrm{kg} \cdot \mathrm{m}^{2}\right)$ & $\mathrm{N} /(\mathrm{kg} \cdot \mathrm{m})$ \\
\hline$\widehat{\theta}_{K x x 1}=640.35$ & $\widehat{\theta}_{K x \theta 1}=48.60$ & $\widehat{\theta}_{K x \theta 1 \star}=42.18$ & $\widehat{\theta}_{K \theta \theta 1}=1124.5$ \\
$\widehat{\theta}_{K x x 2}=167.33$ & $\widehat{\theta}_{K x \theta 2}=23.89$ & $\widehat{\theta}_{K x \theta 2 \star}=25.60$ & $\widehat{\theta}_{K \theta \theta 2}=200.98$ \\
$\widehat{\theta}_{K x x 3}=153.56$ & $\widehat{\theta}_{K x \theta 3}=-572.07$ & $\widehat{\theta}_{K x \theta 3 \star}=48.40$ & $\widehat{\theta}_{K \theta \theta 3}=1108.90$ \\
$\widehat{\theta}_{K y y 1}=725.57$ & $\widehat{\theta}_{K x \theta 4}=-124.38$ & $\widehat{\theta}_{K y \theta 1 \star}=115.03$ & $\widehat{\theta}_{K \theta \theta 4}=467.57$ \\
$\widehat{\theta}_{K y y 2}=172.78$ & $\widehat{\theta}_{K y \theta 1}=74.40$ & $\widehat{\theta}_{K y \theta 2 \star}=-5.25$ & \\
$\widehat{\theta}_{K y y 3}=159.47$ & $\widehat{\theta}_{K y \theta 2}=14.82$ & $\widehat{\theta}_{K y \theta 3 \star}=-7.85$ & \\
& $\widehat{\theta}_{K y \theta 3}=-222.45$ & & \\
& $\widehat{\theta}_{K y \theta 4}=-40.53$ & & \\
\hline
\end{tabular}

Equation (B.2) in the time domain is given by

$$
\begin{gathered}
I_{5}[r]=-\frac{\gamma \Theta_{K}}{\delta^{4} 4 !}\left[\sum_{j=0}^{5}\left(\begin{array}{l}
5 \\
j
\end{array}\right)(-1)^{j}(t-j \delta)^{4} \iota(t-j \delta)\right] \\
+\frac{a_{0} D-a_{1} \gamma \Theta_{K}}{\delta^{4} 3 !}\left[\sum_{j=0}^{5}\left(\begin{array}{l}
5 \\
j
\end{array}\right)(-1)^{j}(t-j \delta)^{3} \iota(t-j \delta)\right] \\
+\frac{D+D_{g}}{\delta^{4} 2 !}\left[\sum_{j=0}^{5}\left(\begin{array}{l}
5 \\
j
\end{array}\right)(-1)^{j}(t-j \delta)^{2} \iota(t-j \delta)\right]
\end{gathered}
$$

where $\iota\left(t-t_{0}\right)$ is the unit step function applied at $t=t_{0}$. The three summations of (B.3) that are inside the brackets are zero at $t=5 \delta$. For instance, the third summation from $t=5 \delta$ to $t=\infty$ is given by

$$
\begin{aligned}
t^{2}-5 & {\left[t^{2}-2 t \delta+\delta^{2}\right]+10\left[t^{2}-4 t \delta+4 \delta^{2}\right] } \\
& -10\left[t^{2}-6 t \delta+9 \delta^{2}\right]+5\left[t^{2}-8 t \delta+16 \delta^{2}\right] \\
& -\left[t^{2}-10 t \delta+25 \delta^{2}\right]=0 .
\end{aligned}
$$

Similarly, the other two summations are zero after $t=5 \delta$. Therefore, $I_{5}[r]$ converges to zero at $t=5 \delta$.

\section{Competing Interests}

The authors declare that there is no conflict of interests regarding the publication of this paper.

\section{Acknowledgments}

Antonio Concha thanks Programa para el Desarrollo Profesional Docente (PRODEP) for supporting this work and also thanks Consejo Nacional de Ciencia y Tecnología (CONACYT) for the financial support given during his postdoctoral stay at the UNAM. The authors also thank UNAMDGAPA PAPIIT for supporting this research through Grants IN109414 and IN109316.

\section{References}

[1] Y. Li and S. T. Mau, "Case study of MIMO system identification applied to building seismic records," Earthquake Engineering and Structural Dynamics, vol. 20, no. 11, pp. 1045-1064, 1991.

[2] J.-M. Ueng, C.-C. Lin, and P.-L. Lin, "System identification of torsionally coupled buildings," Computers and Structures, vol. 74, no. 6, pp. 667-686, 2000.

[3] C. C. Lin, L. L. Hong, J. M. Ueng, K. C. Wu, and C. E. Wang, "Parametric identification of asymmetric buildings from earthquake response records," Smart Materials and Structures, vol. 14, no. 4, pp. 850-861, 2005.

[4] R. D. Nayeri, S. F. Masri, R. G. Ghanem, and R. L. Nigbor, "A novel approach for the structural identification and monitoring of a full-scale 17-story building based on ambient vibration measurements," Smart Materials and Structures, vol. 17, no. 2, Article ID 025006, 2008.

[5] H. Luş, R. Betti, and R. W. Longman, "Identification of linear structural systems using earthquake-induced vibration data," 
Earthquake Engineering \& Structural Dynamics, vol. 28, no. 11, pp. 1449-1467, 1999.

[6] G. Hegde and R. Sinha, "Parameter identification of torsionally coupled shear buildings from earthquake response records," Earthquake Engineering and Structural Dynamics, vol. 37, no. 11, pp. 1313-1331, 2008.

[7] E. Antonacci, A. De Stefano, V. Gattulli, M. Lepidi, and E. Matta, "Comparative study of vibration-based parametric identification techniques for a three-dimensional frame structure," Structural Control and Health Monitoring, vol. 19, no. 5, pp. 579608, 2012.

[8] M. A. M. Torkamani and A. K. Ahmadi, "Stiffness identification of two- and three-dimensional frames," Earthquake Engineering \& Structural Dynamics, vol. 16, no. 8, pp. 1157-1176, 1988.

[9] R. Omrani, R. E. Hudson, and E. Taciroglu, "Story-by-story estimation of the stiffness parameters of laterally-torsionally coupled buildings using forced or ambient vibration data: I. Formulation and verification," Earthquake Engineering \& Structural Dynamics, vol. 41, no. 12, pp. 1609-1634, 2012.

[10] J.-F. Wang, C.-C. Lin, G.-L. Lin, and C.-H. Yang, "Story damage identification of irregular buildings based on earthquake records," Earthquake Spectra, vol. 29, no. 3, pp. 963-985, 2013.

[11] R. Omrani, R. E. Hudson, and E. Taciroglu, "Parametric identification of nondegrading hysteresis in a laterally and torsionally coupled building using an unscented Kalman filter," Journal of Engineering Mechanics, vol. 139, no. 4, pp. 452-468, 2013.

[12] R. Omrani, R. E. Hudson, and E. Taciroglu, "Identification of mass eccentricities from seismic response of three-dimensional buildings," in Proceedings of the 10th International Conference on Urban Earthquake Engineering (CUEE '13), pp. 417-428, ASCE, Tokyo, Japan, March 2013.

[13] J. M. Angeles-Cervantes and L. Alvarez-Icaza, "Identification of seismically excited buildings with two orthogonal horizontal components," Journal of Vibration and Control, vol. 17, no. 6, pp. 881-901, 2011.

[14] A. Yanik, U. Aldemir, and M. Bakioglu, "A new active control performance index for vibration control of three-dimensional structures," Engineering Structures, vol. 62-63, pp. 53-64, 2014.

[15] S. V. Mevada and R. S. Jangid, "Seismic response of torsionally coupled building with passive and semi-active stiffness dampers," International Journal of Advanced Structural Engineering, vol. 7, no. 1, pp. 31-48, 2015.

[16] S. Paul, W. Yu, and X. Li, "Recent advances in bidirectional modeling and structural control," Shock and Vibration, vol. 2016, Article ID 6275307, 17 pages, 2016.

[17] A. Chopra, Dynamics of Structures: Theory and Applications to Earthquake Engineering, Prentice Hall, New York, NY, USA, 2001.

[18] M. Paz and W. Leigh, Structural Dynamics: Theory and Computation, Springer Science+Business media, New York, NY, USA, 5th edition, 2004.

[19] A. Concha, L. Alvarez-Icaza, and R. Garrido, "Simultaneous parameter and state estimation of shear buildings," Mechanical Systems and Signal Processing, vol. 70-71, pp. 788-810, 2016.

[20] S. A. Anagnostopoulos, M. T. Kyrkos, and K. G. Stathopoulos, "Earthquake induced torsion in buildings: critical review and state of the art," Earthquake and Structures, vol. 8, no. 2, pp. 305377, 2015.

[21] H. P. Gavin, R. Morales, and K. Reilly, "Drift-free integrators," Review of Scientific Instruments, vol. 69, no. 5, pp. 2171-2175, 1998.
[22] C. L. Kan and A. K. Chopra, "Elastic earthquake analysis of torsionally coupled multistorey buildings," Earthquake Engineering \& Structural Dynamics, vol. 5, no. 4, pp. 395-412, 1977.

[23] Y.-B. Yang and W.-H. Huang, "Seismic response of light equipment in torsional buildings," Earthquake Engineering and Structural Dynamics, vol. 22, no. 2, pp. 113-128, 1993.

[24] M. D. Trifunac, "Comparisons between ambient and forced vibration experiments," Earthquake Engineering \& Structural Dynamics, vol. 1, no. 2, pp. 133-150, 1972.

[25] S. Sagara and Z.-Y. Zhao, "Numerical integration approach to on-line identification of continuous-time systems," Automatica, vol. 26, no. 1, pp. 63-74, 1990.

[26] T. Söderström and P. Stoica, System Identification, Prentice Hall, New York, NY, USA, 1989. 


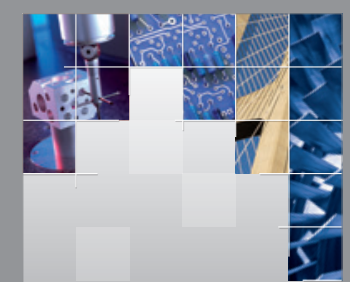

\section{Enfincering}
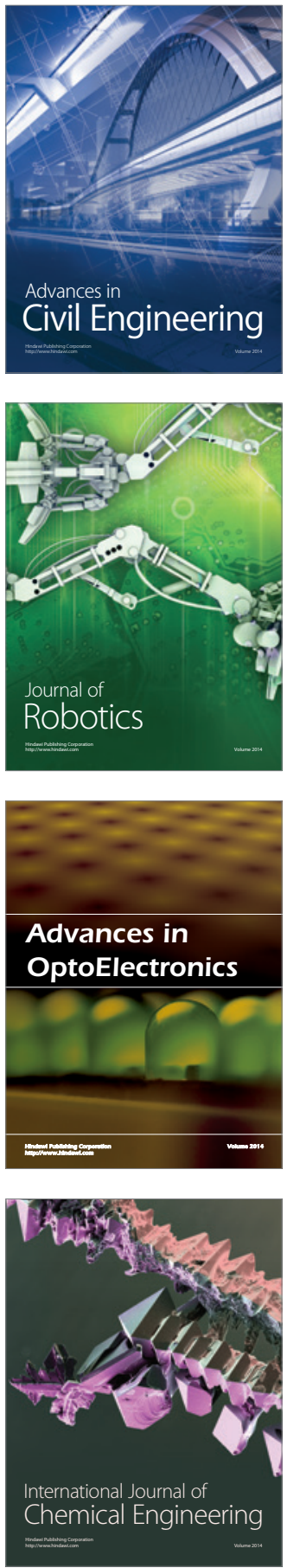

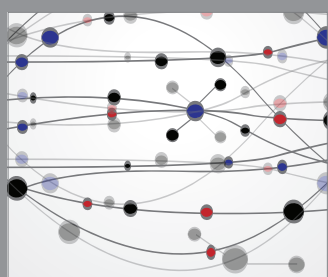

The Scientific World Journal

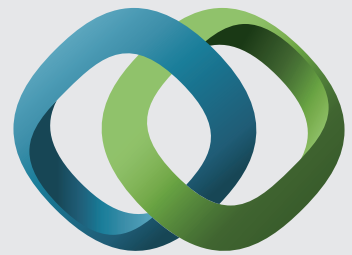

\section{Hindawi}

Submit your manuscripts at

http://www.hindawi.com
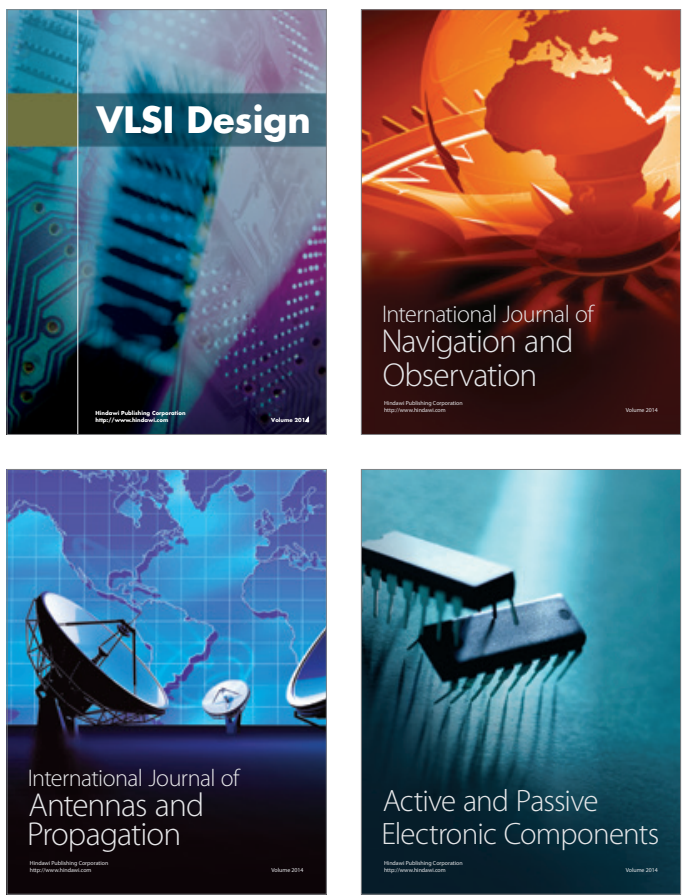
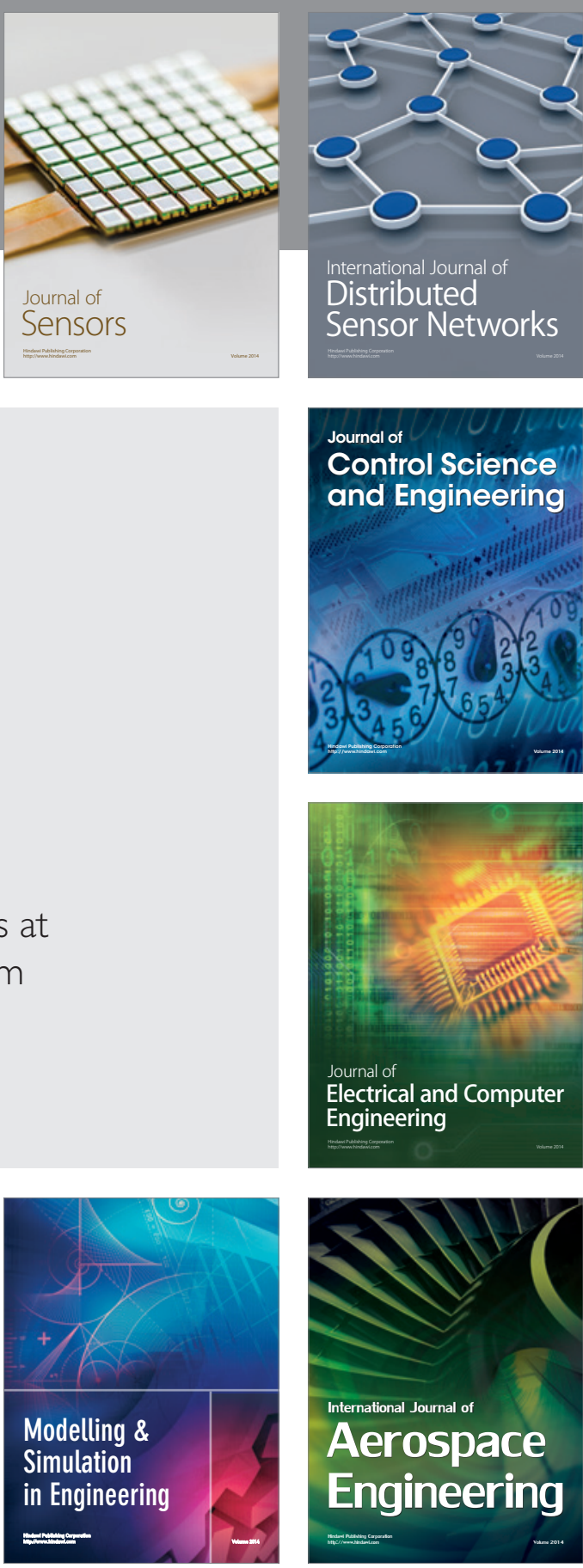

International Journal of

Distributed

Sensor Networks

Journal of

Control Science

and Engineering
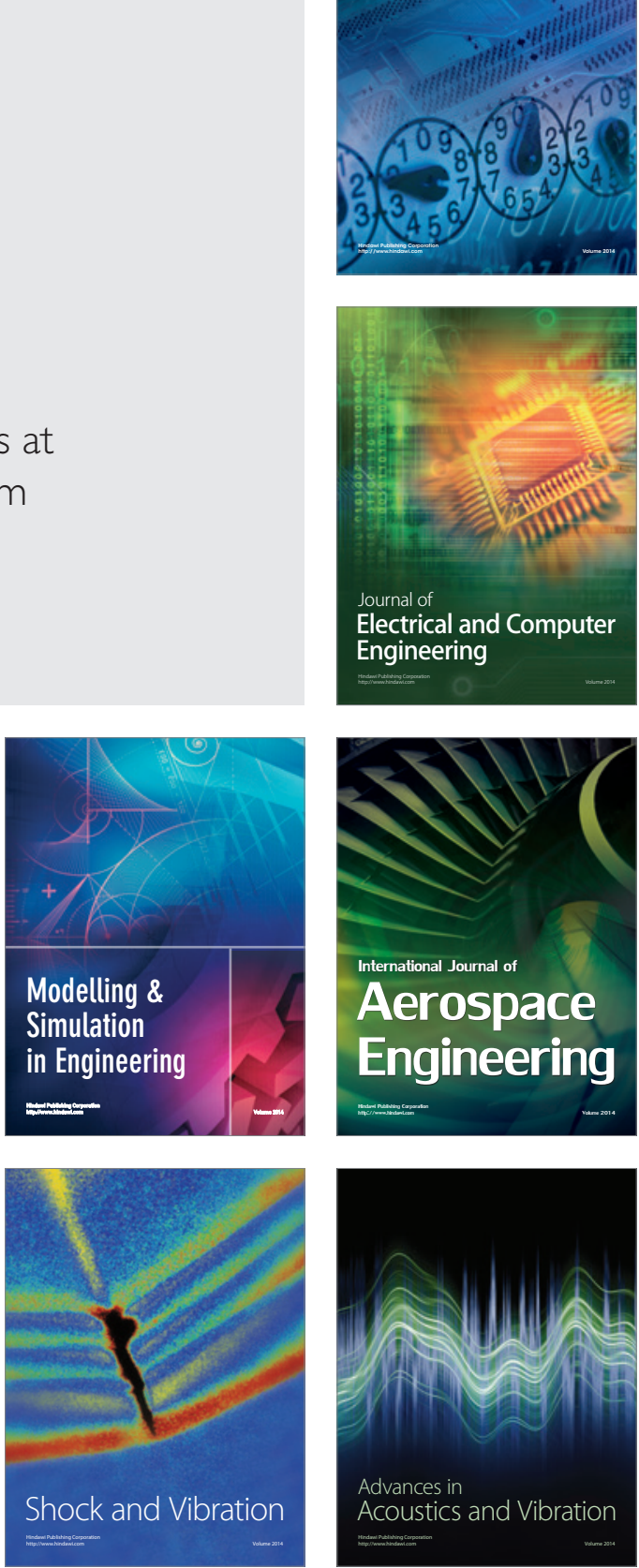